\title{
OPEN Arabidopsis REI-LIKE proteins activate ribosome biogenesis during cold acclimation
}

\author{
Bo Eng Cheong ${ }^{1,2}$, Olga Beine-Golovchuk ${ }^{3,4}$, Michal Gorka ${ }^{3}$, William Wing $\mathrm{Ho} \mathrm{Ho}^{1}$, \\ Federico Martinez-Seidel ${ }^{1,3}$, Alexandre Augusto Pereira Firmino ${ }^{3}$, Aleksandra Skirycz ${ }^{3}$, \\ Ute Roessner ${ }^{1} \&$ Joachim Kopka ${ }^{3 \rrbracket}$
}

Arabidopsis REIL proteins are cytosolic ribosomal 60S-biogenesis factors. After shift to $10^{\circ} \mathrm{C}$, reil mutants deplete and slowly replenish non-translating eukaryotic ribosome complexes of root tissue, while controlling the balance of non-translating 40S- and 60S-subunits. Reil mutations respond by hyper-accumulation of non-translating subunits at steady-state temperature; after cold-shift, a $\mathrm{KCl}$-sensitive $80 \mathrm{~S}$ sub-fraction remains depleted. We infer that Arabidopsis may buffer fluctuating translation by pre-existing non-translating ribosomes before de novo synthesis meets temperatureinduced demands. Reil1 reil2 double mutants accumulate 43S-preinitiation and pre-60S-maturation complexes and alter paralog composition of ribosomal proteins in non-translating complexes. With few exceptions, e.g. RPL3B and RPL24C, these changes are not under transcriptional control. Our study suggests requirement of de novo synthesis of eukaryotic ribosomes for long-term cold acclimation, feedback control of NUC2 and elF3C2 transcription and links new proteins, AT1G03250, AT5G60530, to plant ribosome biogenesis. We propose that Arabidopsis requires biosynthesis of specialized ribosomes for cold acclimation.

The Arabidopsis thaliana Col-0 (Arabidopsis) REI1-LIKE (REIL) proteins, REIL1 (At4g31420) and REIL2 (At2g24500) are homologs of the yeast proteins Rei1 (YBR267W) and its paralog Reh1 (YLR387C). In yeast, the Reil and Reh1 proteins function as ribosome biogenesis factors that participate either in parallel or sequentially in late ribosome biogenesis steps of cytoplasmic $60 \mathrm{~S}$ ribosomal subunit maturation ${ }^{1,2}$. Aside from this function, these proteins are required to maintain growth at suboptimal or cold temperatures. The yeast $\Delta$ reil mutant is cold sensitive already at moderately suboptimal temperatures. The $\Delta$ reil $\Delta$ reh 1 double mutant is even more cold sensitive, while the $\Delta r e h 1$ mutation alone has no effect on growth in the cold ${ }^{3-5}$. Heterologous expression of Arabidopsis REIL1, but not of REIL2 in part complements the cold sensitivity of the yeast $\Delta$ reil mutant ${ }^{6}$. The Arabidopsis REIL paralogs differ in structure from their yeast homologs. REIL1 and REIL2 are four zinc finger proteins with $\mathrm{C} 2 \mathrm{H} 2$ zinc finger domains arranged in two pairs, namely ZF1/ZF2 at the N-terminus and ZF3/ ZF4 at the center of the primary structure ${ }^{6}$. In contrast, both yeast paralogs contain only three canonical zinc fingers ${ }^{1,3}$. This observation is in line with a preliminary phylogenetic study ${ }^{6}$, as both analyses indicate that the duplications of the yeast and Arabidopsis REIL paralogs are evolutionary independent ${ }^{6}$.

Intriguingly, transfer-DNA (T-DNA) mutants of the Arabidopsis REIL1 and REIL2 genes, reil1-1, and allelic reil2-1 or reil2-2, compared to the yeast $\Delta$ reil and $\Delta$ reh1 mutants have convergent growth responses at suboptimal temperature ${ }^{6,7}$. Arabidopsis REIL paralogs are required for plants to grow in the cold, e.g. at $4{ }^{\circ} \mathrm{C}$ or at $10^{\circ} \mathrm{C}$, but not for growth at optimized $20^{\circ} \mathrm{C}$ temperature ${ }^{6,8}$. In agreement with these findings, the identical T-DNA insertion mutants of reil 2 were recently discovered in a screen for chilling sensitive Arabidopsis mutants ${ }^{9}$, namely reil2.1/ stch4-2 (GK_166C10) and reil2.2/ stch4-1 (SALK_040068).

The reil1-1 reil2-1 double mutant, like yeast $\Delta$ reil $\Delta$ reh 1 has a more severe cold phenotype and all but stops development after germination at $10{ }^{\circ} \mathrm{C}$ prior to the emergence of the first rosette leaf ${ }^{6,8}$. The double mutation is, however, non-lethal and a valid system for the investigation of REIL functions in plants as it maintains cellular integrity and acquires freezing tolerance after shifts from 20 to $4{ }^{\circ} \mathrm{C}$ or $10{ }^{\circ} \mathrm{C}$ cold ${ }^{8}$. Expression of amino-terminal FLUORESCENT PROTEIN (FP)-REIL fusion proteins driven by the UBIQUITIN10 promoter rescue the mutant

\footnotetext{
${ }^{1}$ School of BioSciences, The University of Melbourne, Victoria 3010, Australia. ${ }^{2}$ Biotechnology Research Institute, Universiti Malaysia Sabah, Jalan UMS, 88400 Kota Kinabalu, Sabah, Malaysia. ${ }^{3}$ Max-Planck-Institute of Molecular Plant Physiology, Am Mühlenberg 1, 14476 Potsdam-Golm, Germany. ${ }^{4}$ Biochemie-Zentrum, Nuclear Pore Complex and Ribosome Assembly, Heidelberg University, Im Nevenheimer Feld 328, 69120 Heidelberg, Germany. ${ }^{\square}$ email: Kopka@mpimp-golm.mpg.de
} 
phenotypes $^{8}$. The two allelic reil2-1 and reil2-2 mutants are both growth retarded and form small spoon-shaped leaves at $10^{\circ} \mathrm{C}^{6,9}$. This phenomenon reverts after shift from cold to optimal $20^{\circ} \mathrm{C}$. Except for slightly delayed germination, the reil1-1 mutant, similar to yeast $\Delta$ reh1, has no growth phenotype under the currently tested low temperature conditions $s^{6,8}$.

Previous functional analyses of Arabidopsis REIL proteins focused on vegetative photoautotrophic plant rosettes that were cultivated on soil and acclimated to $10^{\circ} \mathrm{C}$ temperature. Conserved functions of the Arabidopsis REIL proteins and new functions that evolved on the path towards multicellular embryophyte plants became apparent ${ }^{8}$. As was expected in analogy to yeast, FP-REIL1 and FP-REIL2 fusion proteins localize to the cytosol ${ }^{8,9}$ and the REIL protein, appear to interact with eukaryotic ribosome complexes containing the 60S large ribosome subunit (LSU) ${ }^{8-10}$. Our current study locates REIL2 to the non-translating 60 S fraction of Col-0 wild type roots.

In rosette leaves, REIL proteins accelerate $10^{\circ} \mathrm{C}$ cold induced accumulation of cytosolic ribosome subunits and of cytosolic ribosomal RNA (rRNA). After cold shift, REIL proteins enhance gene expression of structural proteins of cytosolic ribosomes, ribosome biogenesis factors, and cytosolic translation initiation or elongation factors $^{8}$. The acclimation responses occur within the first week after cold shift, while Arabidopsis wild type plants pass through a lag-phase before resuming growth. All of these processes lag behind in the reil1-1 reil2-1 mutant that does not resume growth in the cold ${ }^{6}$. Our current study confirms these observations in roots and provides mechanistic insights beyond. Besides an influence on cold-induced plant ribosome remodeling and on the accumulation of cytosolic ribosome subunits after cold shift, Arabidopsis REIL proteins are apparently involved in plant-specific processes. For example, the reil1-1 reil2-1 mutant does not activate FLOWERING LOCUS T gene expression in mature leaves after the cold shift and exhibits plant cold-acclimation responses at the $20^{\circ} \mathrm{C}$ non-acclimated state, including premature activation of the C-REPEAT/DRE BINDING FACTOR1/ DEHYDRATION-RESPONSIVE ELEMENT BINDING (CBF/ DREB) regulon ${ }^{8}$. Analyses of the single paralog reil2/ stch4 mutants confirmed the interaction of reil2 function with the CBF/ DREB regulon ${ }^{11-13}$, with REIL2 deficiency delaying CBF/ DREB regulon activation and reducing $\mathrm{CBF} / \mathrm{DREB}$ protein accumulation in the cold. Ectopic overexpression of REIL2 under control of the 35 S promoter conveyed enhanced chilling and freezing tolerance under mixotrophic in vitro conditions ${ }^{9}$.

To reveal organ-independent and likely direct or primary functions of Arabidopsis REIL proteins from more indirectly associated responses to REIL deficiency, we analyzed the root system of Arabidopsis Col-0 wild type and of reil mutant plants under $20^{\circ} \mathrm{C}$ to $10^{\circ} \mathrm{C}$ cold shift conditions and compared these results to previous analyses of mutant and wild type Arabidopsis rosette leaves. To obtain sufficient amounts of root material for multi-level systems analyses, we changed our experimental conditions from soil-grown plants ${ }^{8}$ to a mixotrophic hydroponic system that meets these demands. In this cultivation system, reil mutants retained reduced growth, but attenuated the previously reported strong growth and developmental phenotypes. For our current study, we did not adjust the growth conditions to stronger phenotypes. By analyzing reil mutants under conditions that cause small, but still noticeable phenotypes, we hope to reveal mutant effects that otherwise may be obscured by pleiotropic and secondary responses. For the purposes of identifying and understanding the mechanism of ribosome biogenesis in which Arabidopsis REIL proteins are involved and of discovering potential roles of REIL proteins for plant ribosome heterogeneity ${ }^{14}$, we analyzed ribosomal complexes and applied transcriptome and proteome profiling methods. In addition to the previously reported double mutant reil1-1 reil2- $1^{6-8}$, we introduce a second double mutant, reil1-1 reil2-2, to our studies and analyzed abundances of ribosomal complexes from the single-paralog mutant lines, reil1-1, reil2-1 and reil2-2, from which the double mutants originate.

\section{Results}

Hydroponic growth of reil mutant plants compared to the Arabidopsis Col-0 wild type. The established hydroponic system ${ }^{10}$ enabled sterile photomixotrophic cultivation of up to four Arabidopsis Col-0 wild type or mutant plants within a single container with $2 \%(\mathrm{w} / \mathrm{v})$ sucrose in a liquid growth medium ${ }^{15}$ (Supplementary Fig. S1). Photoautotrophic cultivation of reil1 reil2 double mutants (DKOs) without sucrose caused slow growth and generated early flowering, dwarfed plants with small root systems. For this reason, we chose photomixotrophic cultivation for the current study. In the presence of $2 \%$ (w/v) sucrose, the Arabidopsis wild type reached the 10-leaf stage, i.e. Arabidopsis stage $1.10^{16}$, after approximately 4 weeks under standard long-day photoperiod conditions with external temperatures set to $20^{\circ} \mathrm{C}$ during the day and $18{ }^{\circ} \mathrm{C}$ during night (Supplementary Fig. S1). Morphology of Arabidopsis rosettes differed from soil-grown plants. The leaf petioles were elongated and leaf laminae smaller than on soil ${ }^{6,8}$. Leaves of individual stage 1.10 plants overlapped minimally. From this stage onward, the single root systems entangled with each other and in part with the mesh that was required for cultivation. Only harvests of the complete root systems from four plants of a single container were feasible and allowed rapid physiological inactivation (Supplementary Fig. S2). For this reason, all biological replicates were pooled root samples of at least four plants from single growth containers or of more plants from multiple containers.

Root and shoot growth of the Arabidopsis wild type continued after cold shift. Cold shift was at stage 1.10 and exposed plants to $10{ }^{\circ} \mathrm{C}$ during the day and $8{ }^{\circ} \mathrm{C}$ during night under otherwise unchanged conditions. Plants phase-shifted to the generative phase and produced small inflorescences between 7 and 21 days after exposure to cold (Supplementary Fig. S2). Photomixotrophic cultivation did not fully abolish differential growth of mutants and Col-0 but growth and development of the reil1-1 mutant was similar to wild type. Shoots of the mutants, reil2-1, reil2-2, and the double mutants, reil1-1 reil2-1 (DKO1) and reil1-1 reil2-2 (DKO2) remained slightly smaller than wild type after cold shift, but all mutants continued to grow and developed inflorescences (Supplementary Fig. S2). Shift to low temperature induced spoon-shaped leaf morphology of reil2 mutants, but this phenotype was strongly attenuated compared to soil-grown reil2 mutants ${ }^{6}$. In contrast to soil cultivation ${ }^{8}$, 
the double mutants were not fully growth arrested after $10^{\circ} \mathrm{C}$ cold shift in our hydroponic growth system and continued to grow.

A preliminary study of mutant root systems from single plants of our cultivation system (Supplementary Fig. S3) showed delayed root growth and a shortened acropetal part of the primary root of the double mutants compared to the wild type. These observations became apparent 7 to 21 days after cold shift. At 21 days after cold shift, double mutant and reil1-1 roots appeared to be less branched, indicative of an altered root branching pattern compared to the wild type (Supplementary Fig. S3). Further characterization of altered root growth and branching pattern were not performed due to the limitations of the cultivation system. We chose standardized temperature shift experiments that were similar in experimental design to previous experiments with soil-grown plants $^{6,8}$. In agreement with these experiments, we started sampling at developmental stage 1.10 with the nonacclimated state followed by sampling at $1,3,7$, and 21 days after shift to $10^{\circ} \mathrm{C}$ cold.

Analyses of ribosome complex abundances from roots confirm association of REIL deficiency with delayed 605 LSU accumulation after cold shift. Previous analyses of cytosolic ribosome complexes from Arabidopsis showed activation of cytosolic rRNA expression and concerted accumulation of transcripts coding for cytosolic ribosomal proteins (RPs) after a $10{ }^{\circ} \mathrm{C}$ cold shift from optimized temperatures ${ }^{8}$. The reil1-1 reil2-1 mutant delayed these responses and the accumulation of the 60S LSU fraction compared to wild type. In this previous study ${ }^{8}$, we analyzed leaf material of soil-cultivated rosette plants. The presence of chloroplast ribosomes interfered in parts with the abundance analysis of cytosolic ribosome complexes. We succeeded to separate a 60S LSU fraction from the chloroplast 50S LSU and chloroplast 70S from 80S monosomes, but the polysome fractions or chloroplast $30 \mathrm{~S}$ and cytosolic $40 \mathrm{~S}$ small subunits (SSUs) remained non-resolved. For this reason, we did not investigate previously, an indicated concerted regulation of cytosolic 60S LSU with the abundances of other cytosolic ribosomes complexes.

To overcome this limitation and to extend and validate our analyses of the delayed accumulation of the 60S LSU in REIL deficient mutants, we focused on root material that became available in sufficient amounts of $\sim 100 \mathrm{mg}$ fresh weight per analysis by hydroponic cultivation. As we expected, plastid ribosome complexes, 30S, 50S, and 70S, were negligible in this material and much less abundant than cytosolic ribosomes. We obtained improved separation and purity of 40S SSU, 60S LSU, 80S ribosome fractions and analyzed the abundance of these fractions in wild type compared to the double mutants, reil1-1 reil2-1 and reil1-1 reil2-2, and the respective singleparalog mutants, reil1-1, reil2-1, and reil2-2 (Fig. 1). Based on the equal amounts of root fresh weight Arabidopsis wild type accumulated cytosolic ribosome complexes, especially the $80 \mathrm{~S}$ fraction, at $1,3,7$, and 21 days after shift to $10^{\circ} \mathrm{C}$. Profiles of ribosome complexes from both, the reil1-1 reil2-1 and the reil1-1 reil2-2 mutant, were distinct from wild type. The abundance of the 60S LSU fraction of both double mutants approximately equaled the $80 \mathrm{~S}$ fraction already in the non-acclimated state. This difference to wild type persisted throughout cold acclimation (Fig. 1). In agreement with previous observations from leaf material, 60S LSU accumulation after cold shift lagged behind wild type in both reil double mutants (Fig. 1).

Changes of ribosome complex abundances reveal inter-complex correlations and responses to REIL deficiency. This observation and associated effects of other ribosome fractions became apparent by analyzing $\log _{2}$-transformed ratios of ribosome complex abundances of mutants compared to wild type at each time point of our temperature shift experiment (Fig. 2, Supplemental Table S1). In the non-acclimated state, all reil mutants increased the $60 \mathrm{~S}$ LSU and the $40 \mathrm{~S}$ SSU fractions $\sim$ two-fold relative to wild type (Fig. $2 \mathrm{~A}, \mathrm{~B}$ ), but $80 \mathrm{~S}$ monosomes had wild type levels (Fig. 2C). These observations in the non-acclimated state coincided with a slight increase of sum of all detected ribosome complexes (Fig. 2D). At 1 day after cold shift, all reil mutants except reil1-1 reduced the 60S LSU and the 40S SSU fractions to approximate wild type levels (Fig. 2A,B). Reductions of these fractions were only marginal in the reil1-1 mutant. All mutants subsequently returned to at least wild type levels and in most cases approximated the initial over-accumulation of 60S LSUs and 40S SSUs. The reil1-1 reil2-1 and the reil1-1 reil2-2 double mutants reduced the abundance of the 80S fraction up to four-fold relative to wild type upon cold shift (Fig. 2C). This phenomenon occurred at 1 day after cold shift and continued during prolonged cold exposure. In the reil2 single-paralog mutants, the relative abundances of the $80 \mathrm{~S}$ fraction were similarly but less reduced. The sum of all ribosome complexes was reduced at day 1 and 21 after cold shift in the double mutants and in part in the single mutants (Fig. 2D). The changes of 60S LSU and the 40S SSU abundances correlated with a Pearson's correlation coefficient of $r=0.945$ across all measurements of our experiment (Fig. 3A). The relative abundances of the 60S LSU and 80S monosome fractions, however, correlated less stringently, $r=0.607$ (Fig. 3B).

The effect of the double mutations on the $80 \mathrm{~S}$ fraction was the largest and most consistent among the observed changes of cytosolic ribosome complexes. We exemplarily tested the $80 \mathrm{~S}$ fractions of wild type and the reil1-1 reil2-1 mutant for previously described heterogeneity ${ }^{17,18}$ using a sucrose sedimentation gradient tuned to separate translating ribosome fractions, specifically the $80 \mathrm{~S}$ monosomes from the polysomes. Translating yeast or mammalian ribosomes are stable at high ionic strength. A sub-fraction of monosomes that are thought to be non-translating can be dissociated in vitro by elevating $\mathrm{KCl}$ levels during preparation ${ }^{17,18}$. Like in yeast and mammals, the $80 \mathrm{~S}$ monosomes of plant roots partitioned into a $\mathrm{KCl}$-sensitive and a stable fraction (Fig. 4 ). $\mathrm{KCl}$ concentrations that were elevated from regular $200 \mathrm{mM}$ to $400 \mathrm{mM}$ did not affect the root polysome fraction. In contrast, the $80 \mathrm{~S}$ fraction that accumulated in Arabidopsis wild type roots at 21 days after cold shift was to a large extend $\mathrm{KCl}$-sensitive as was indicated by associated decreases of $80 \mathrm{~S}$ monosomes and increases of the 60 S LSU and 40S SSU fractions (Fig. 4A). The 80 S fraction of the reill-1 reil2-1 mutant contained only a minor amount of $\mathrm{KCl}$-sensitive $80 \mathrm{~S}$ monosomes (Fig. 4B). Like wild type, the polysome fraction of the reil1-1 reil2-1 mutant was not $\mathrm{KCl}$-sensitive. Polysome abundance was unchanged or slightly increased relative to wild type. We 


\section{Wild type \\ (Col-0)

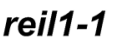 \\ reil2-1 \\ reil2-2 \\ reil1-1 reil 2-1 reil1-1 reil 2-2}

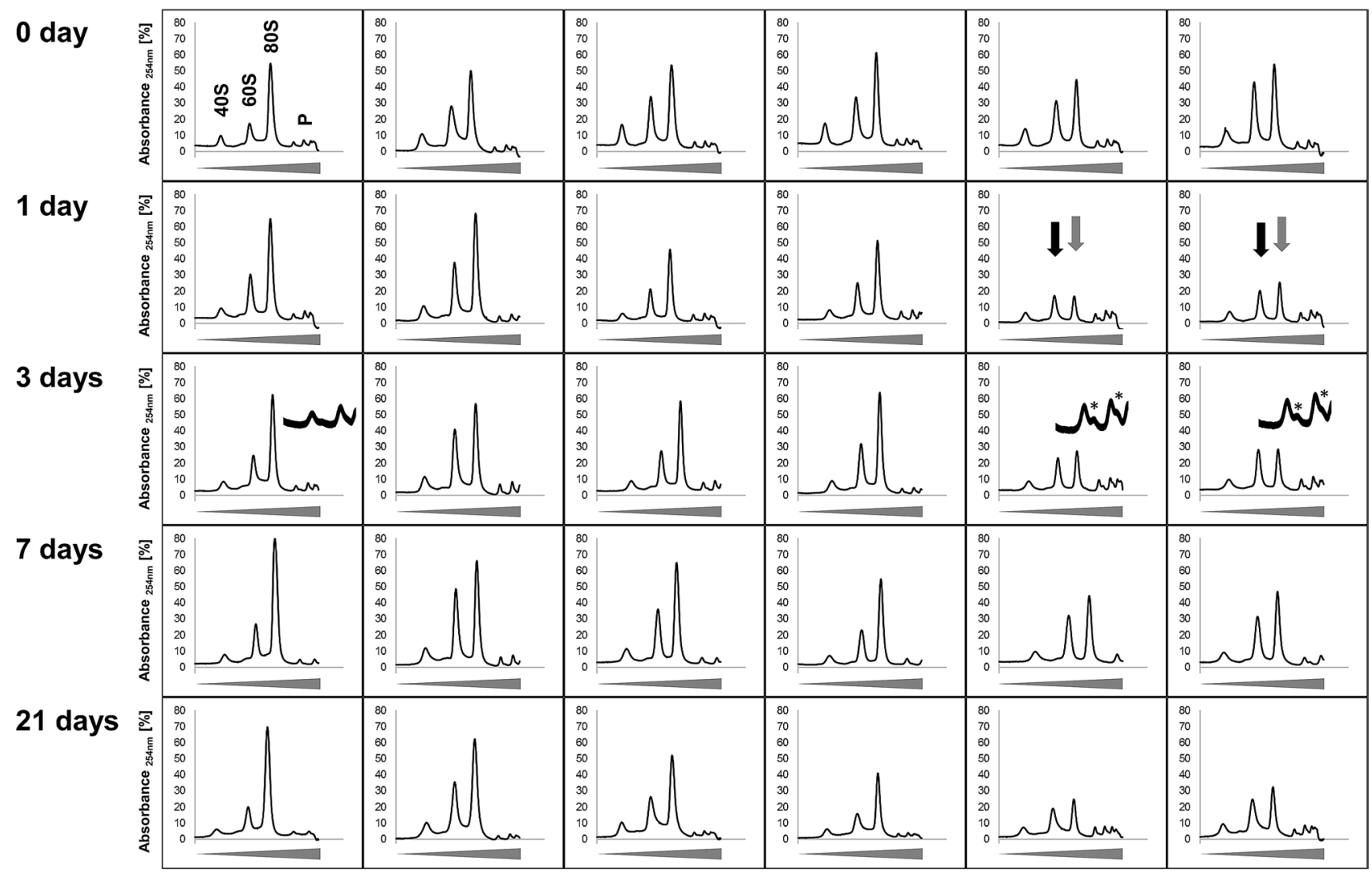

Figure 1. Sucrose density gradient analyses of ribosome complexes from equal amounts (fresh weight) of hydroponically grown total root material before ( 0 day) and at $1,3,7$, or 21 days after shift from $20^{\circ} \mathrm{C}$ (day)/ $18^{\circ} \mathrm{C}$ (night) to $10^{\circ} \mathrm{C}$ (day) and $8{ }^{\circ} \mathrm{C}$ (night). The Arabidopsis thaliana wild type (Col-0) was compared to the single-paralog mutants, reil1-1, reil2-1, reil2-2, and to the double mutants, reil1-1 reil2-1 and reil1-1 reil2-2. Absorbance at wavelength $254 \mathrm{~nm}$ was recorded continuously during fractionation of $15-60 \%$ sucrose density gradients (tapered bars) and background corrected by a non-sample control. This is a composite figure of multiple centrifugation runs that each contained a non-sample control. Gradients varied slightly with each centrifugation run. Note the altered abundance pattern of ribosome complexes from the reil1-1 reil2-1 and reil1-1 reil2-2 double mutants in non-acclimated and acclimating states and the delay relative to Col- 0 of $60 \mathrm{~S}$ LSU (black arrows) and 80S monosome (grey arrows) accumulation after cold shift. Inserts into the day 3 analyses magnify the low-oligomer polysome region and demonstrate accumulation of half-mer polysomes $\left({ }^{*}\right)$ in the double mutants early after cold shift. Half-mer polysomes are polysome complexes with a stalled $40 \mathrm{~S}$ preinitiation complex and one or more fully assembled 80 S ribosomes. Positions of $40 \mathrm{~S}, 60 \mathrm{~S}, 80 \mathrm{~S}$ and lowoligomer polysome complexes $(\mathrm{P})$ are indicated in the top left panel.

conclude that the failure of the double mutants to accumulate the $80 \mathrm{~S}$ monosome fraction is likely a deficiency of accumulating the non-translating $80 \mathrm{~S}$ sub-fraction.

In summary, reil mutants appear to compensate deficiency of cytosolic ribosome biogenesis by over accumulation of free 60 S LSU and 40 S SSU and in the case of the double mutants by recruiting non-translating monosomes. These experiments, however, cannot distinguish between over accumulation of functional or incorrectly assembled ribosome subunits.

REIL deficiency deregulates transcriptional temperature acclimation responses in roots. In the following, we focused on the two double mutants, reil1-1 reil2-1 and reil1-1 reil2-2, and characterized samples of non-acclimated ( 0 day) and the early cold-acclimating states at 1 day or at 7 days after cold-shift to Col- 0 wild type as basis of the subsequent ribosome and proteomic studies. Across the complete observation period, the reil1-1 reil2-1 and reil1-1 reil2-2 mutants shared constitutive reduction of expression of both reil genes in roots (Supplemental Fig. S5). The reduced gene expression in roots was similar to or in the case of residual reil2 expression exceeded previous observations of reil2 silencing in reil1-1 reil2-1 leaves ${ }^{8}$, see Supplemental Table S2 of $^{8}$.

We chose the early cold-acclimating states according to our previous observations of a Col-0 growth arrest after cold-shift. This arrest extended up to 7 days before growth resumed in the cold ${ }^{8}$. Thereby, we aimed to avoid, as best as possible, pleiotropic mutant effects that result from differential growth of the reil mutants at later 
A
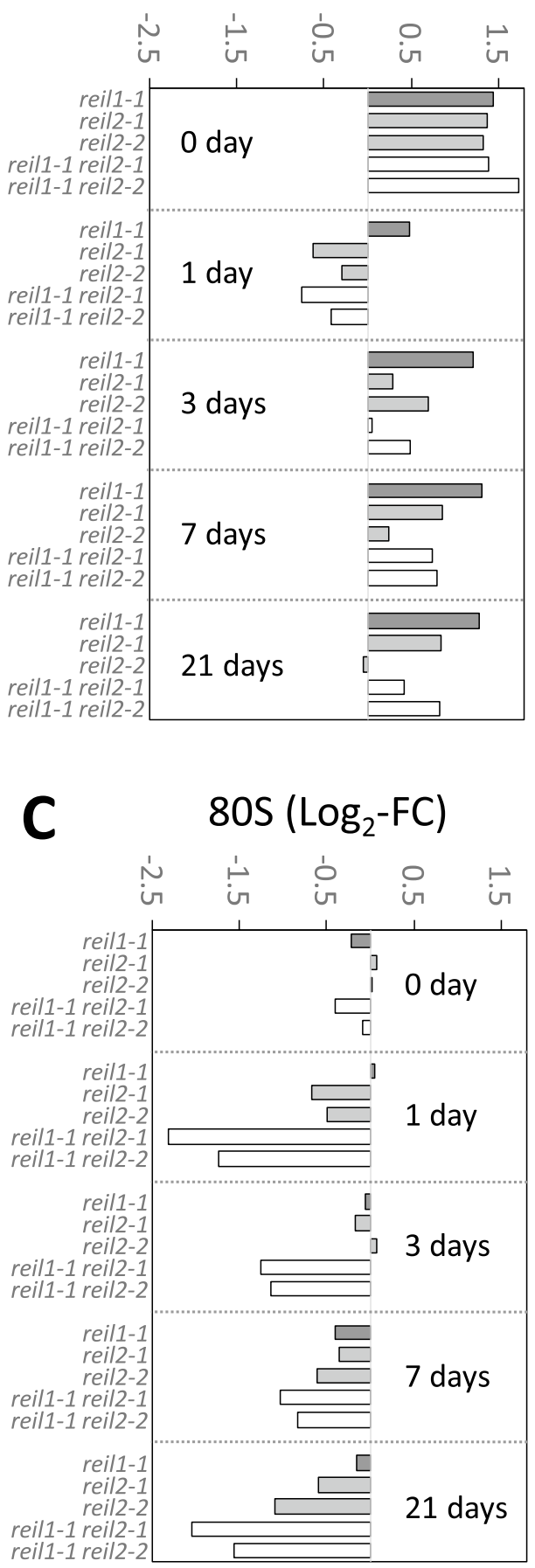

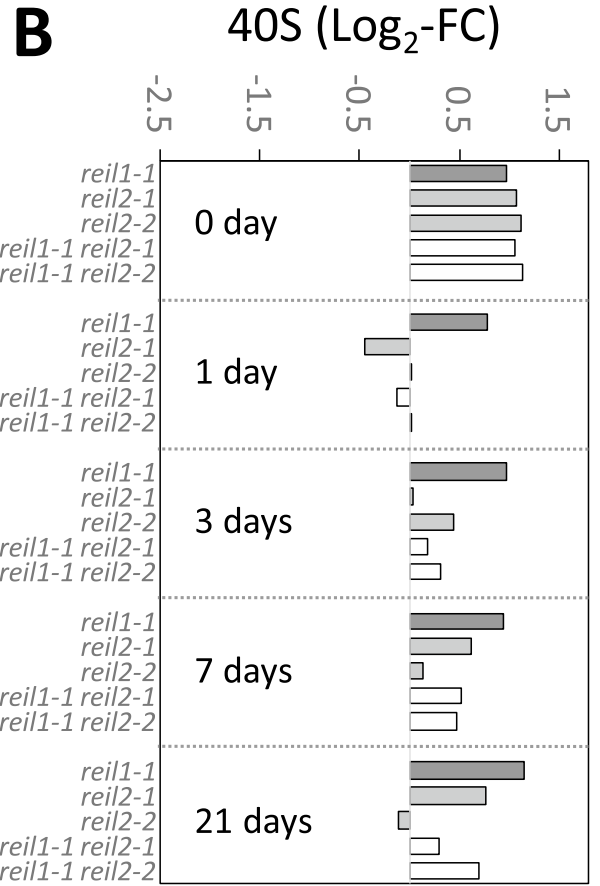

D

Total $\left(\log _{2}-\mathrm{FC}\right)$

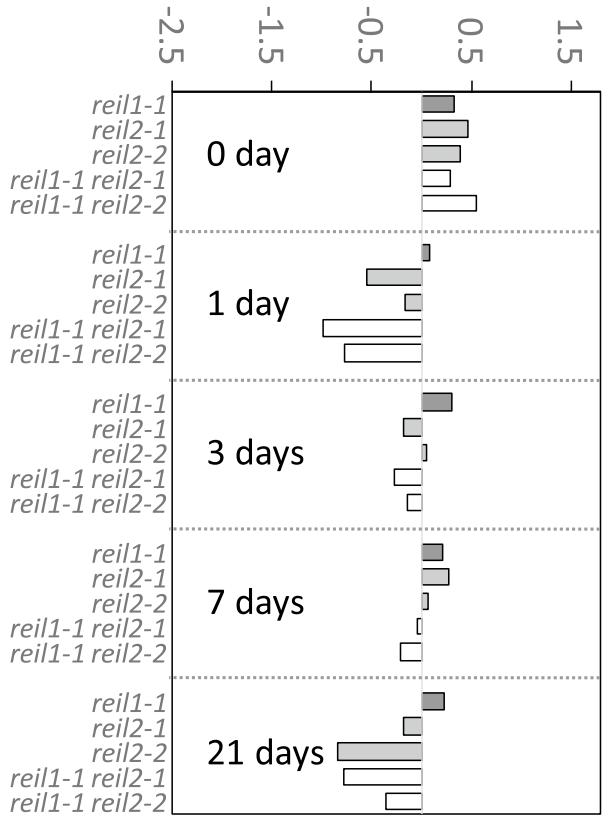

Figure 2. Relative quantification of abundances of ribosome complexes from non-acclimated ( 0 day) and $10^{\circ} \mathrm{C}$ cold acclimating reil mutants at 1, 3, 7, or 21 days after shift (cf. Figure 1). Baseline corrected peak areas of ribosome complexes were integrated and $\log _{2}$-transformed ratios calculated relative to the wild type fractions at each time point, i.e. $\log _{2}$-fold change (FC). The 60S LSU (A), 40S SSU (B), 80S monosomes (C) and the sum ("total") of all detected ribosome complexes (D), were analyzed (dark grey: reil1-1, light grey: reil2-1 and reil2-2, white: double mutants, reil1-1 reil2-1 and reil1-1 reil2-2).

time points. Besides sample characterization, we aimed to reveal at transcriptional level, potential mechanisms that may regulate ribosome complex abundance or may take part in potential compensation responses to reil deficiency (Supplemental Table S2). 

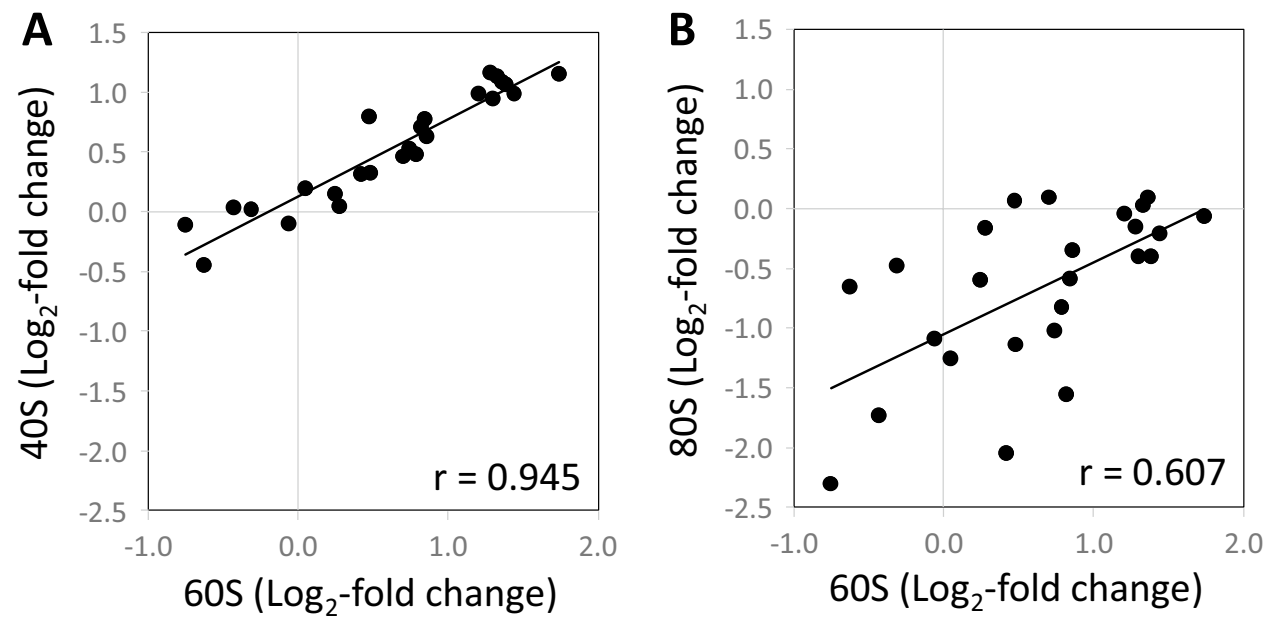

Figure 3. Correlation analyses of $\log _{2}$-transformed ratios of ribosome complex abundances comparing the cold induced changes of abundances relative to wild type of the 40S SSU (A) and of the 80S monosome fractions (B) to the changes of the 60S LSU fraction, respectively, across all analyzed reil mutants (cf. Figure 2). Inserts are the Pearson's correlation coefficients ( $r$ ) assuming a linear trend.

Roots of Col-0 and the reil double mutants responded in our axenic, photomixotrophic, hydroponic growth system to $10^{\circ} \mathrm{C}$ cold shift by expected functional enrichment of transcript changes ${ }^{19}$ that relate to cold stress. Expression of genes belonging to the GOs, cold acclimation (GO:0009631) or cold response (GO:0009409), increased over time (Fig. 5A, Supplemental Table S3). Marker genes of cold acclimation and response, e.g. CBF1, CBF2, CBF3, KIN1, KIN2, VIN3, COR15A or COR15B, changed accordingly (Supplemental Fig. S4). Inversely to the cold response, expression of heat response genes (GO:0009408) significantly decreased in our root samples (Fig. 5A). This observation differed compared to our previous experiments with soil cultivated rosette leaves ${ }^{8}$ and was accompanied by high light intensity (GO:0009644), hydrogen peroxide (GO:0042542) and reactive oxygen species (GO:0000302) responses. Together these observations indicated that the roots of Col-0 and those of the reil double mutants were subject to stress in our cultivation system (Fig. 5A). This stress likely arose due to the artificial, light exposed hydroponic cultivation of roots in this study.

Our previous study showed that mature leaves of reil1-1 reil2-1 are prematurely cold acclimated at $20^{\circ} \mathrm{C}^{8}$. In our current study, we selected GOs that had significant enrichment of $\log _{2}$-FCs in non-acclimated reil double mutant roots at $20^{\circ} \mathrm{C}$ compared to non-acclimated wild type at the same temperature. We compared these changes to the significant $\log _{2}$-FCs of wild type after 7 days at $10^{\circ} \mathrm{C}$ relative to the non-acclimated wild type. For this purpose, we applied two thresholds of FDR-adjusted $P<0.01$ or 0.05 (Fig. 5B,C). The mean $\log _{2}$-FCs of genes from the selected GOs of the non-acclimated reil double mutants appeared to correlate to the mean $\log _{2}$-FCs of the cold acclimated wild type. However, part of the cold responsive GOs responded inversely (Fig. 5B,C). The overlay of both, positive and negative correlations was even more obvious at single gene level (Fig. 5D,E). In all of our four correlation analyses, namely the two double mutants at the two significant thresholds, $P<0.05$ and $P<0.01$, the assumption of linear correlations did not apply. Therefore, correlation coefficients were not calculated. We concluded that reil double mutant roots deregulate transcriptional cold acclimation at $20^{\circ} \mathrm{C}$ in our growth system, with two subsets of genes, which either prematurely activate or prematurely deactivate in our photomixotrophic cultivation system that masks carbohydrate mediated signals. This response differs from premature cold acclimation as was indicated by transcription factors that control cold acclimation, e.g. CBF1, $C B F 2$ and $C B F 3$. Transcripts of these factors did not consistently accumulate prematurely and expression of cold response marker genes, e.g. KIN1, KIN2, VIN3, COR15A, and COR15B, was even reduced (Supplemental Fig. S4).

REIL deficiency affects transcription of root morphogenesis genes and activates expression of cytosolic ribosome and translation related genes. Transcription of $34 \mathrm{GO}$ terms had significant (FDR-adjusted $P<0.05$ ) enrichment in both reil mutants relative to non-acclimated Col-0 across all time points of our study (Supplemental Table S3). These GO terms comprised the four previously mentioned stress stimuli (Fig. 5A) and two miscellaneous GOs, manganese ion binding, GO:0030145, and glycoside metabolic processes, GO:0016137 (Supplemental Fig. S6).

A major common response pattern of the mutants corresponded to our observation of altered mutant root morphology (Supplemental Fig. S3). Twelve GO terms had constitutively reduced expression. These GOs were related to developmental processes, GO:0021700, root and root hair morphogenesis, GO:0010015 and GO:0010054, and cell wall organization and biogenesis, GO:0071554 (Supplemental Fig. S6A).

Eight of the remaining GO terms were related to translation or ribosome complexes, specifically to both subunits of cytosolic ribosomes (Fig. 6A,B). Col-0 transiently activated gene expression of cytosolic ribosomal proteins (RPs) at day 1. In contrast, reill-1 reil2-1 and reill-1 reil2-2 consistently activated expression of cytosolic RP genes both, at days 1 and 7 after the cold shift. Expression of organelle RP genes did not significantly 
A

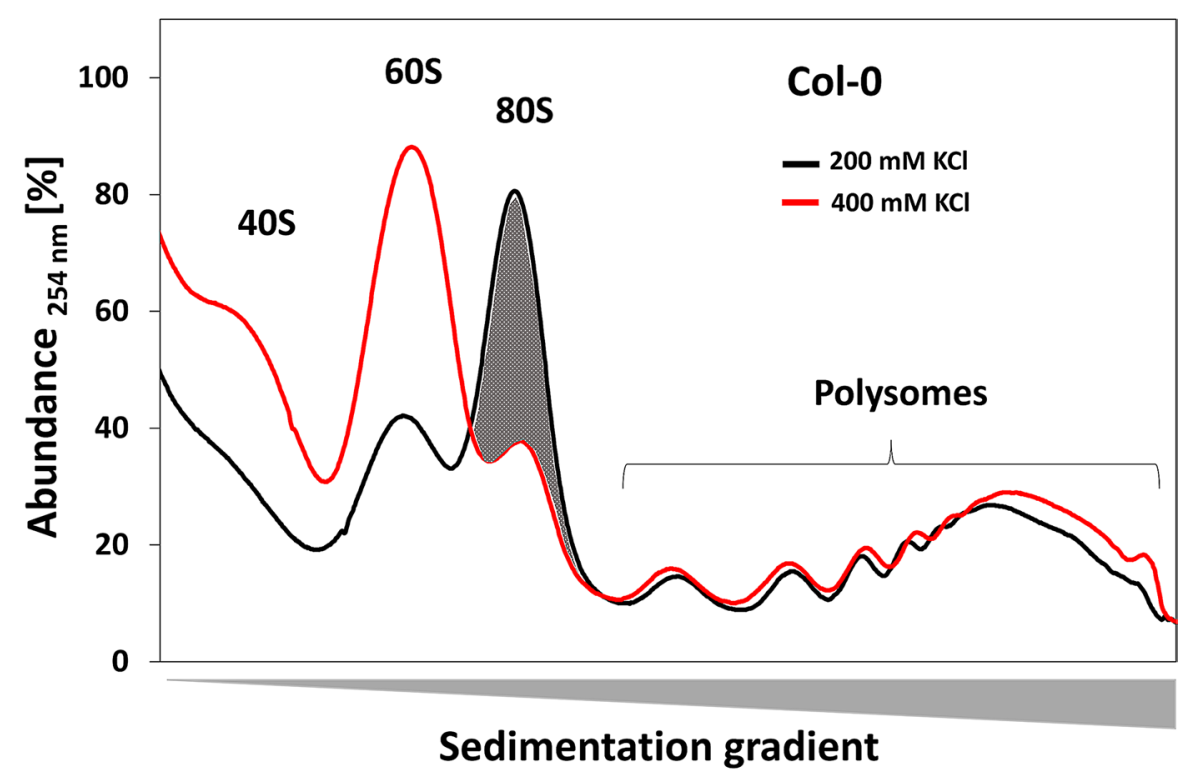

B

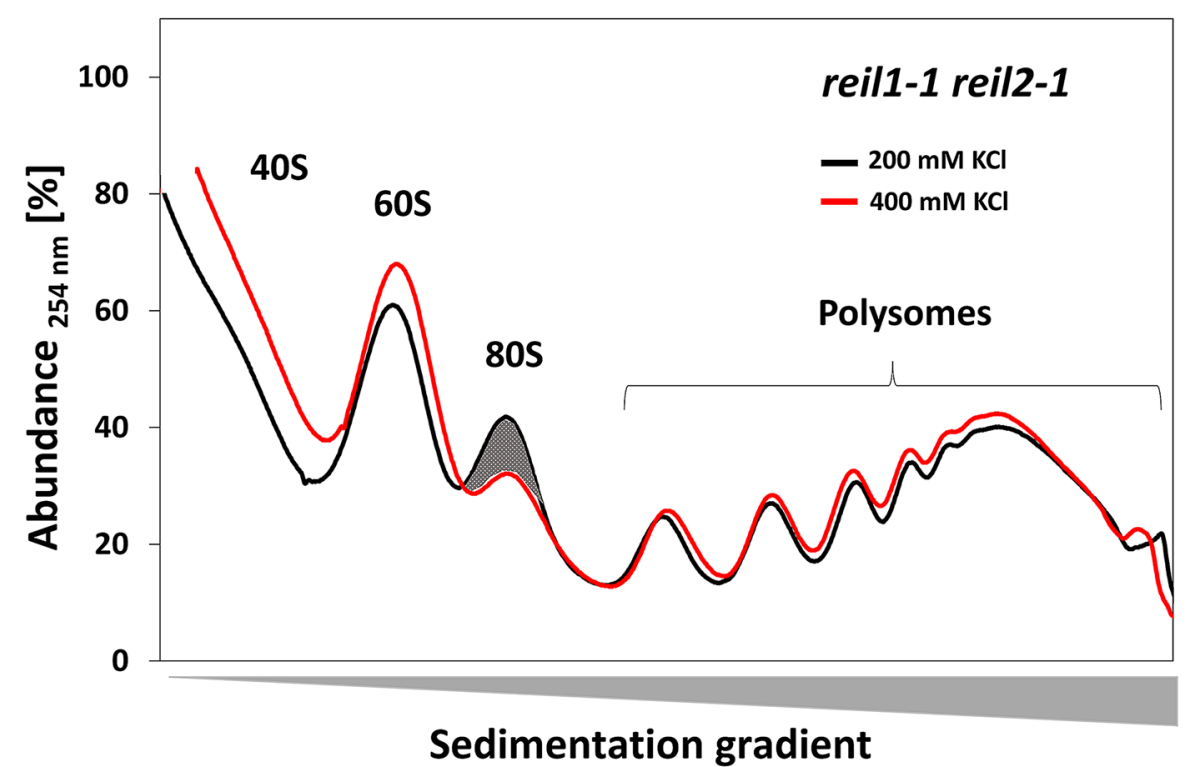

Figure 4. In vitro $\mathrm{KCl}$-sensitivity test of monosomes and polysomes that were prepared from roots sampled at 21 days after start of $10^{\circ} \mathrm{C}$ cold-acclimation. Arabidopsis Col-0 wild type (A) was compared to the reill-1 reil2-1 double mutant (B). Initial samples were homogenized and split into equal technical replicates. These replicates were extracted with $\mathrm{PEB}$ that either, contained a normal $\mathrm{KCl}$ concentration $(200 \mathrm{mM}$, black) or an elevated $\mathrm{KCl}$ concentration ( $400 \mathrm{mM}$, red). Note that the acclimated wild type accumulated a large fraction of $\mathrm{KCl}$-sensitive monosomes, whereas $\mathrm{KCl}$-sensitive monosomes were almost absent from cold acclimated reil1-1 reil2-1 preparations.

change in both mutants (Fig. 6A). The GO terms translation (GO:0006412) and the GO terms of cytosolic RP genes (GO:0022625-GO:0022627) responded similarly. Translation was affected in the sub-categories translation initiation (GO:0003743) and eukaryotic translation initiation factor 3 (eIF3, GO:0005852) (Fig. 6B).

The activation of cytosolic ribosome and translation related gene expression in mutant roots mirrored the effects found in reil1-1 reil2-1 leaves ${ }^{8}$. Kinetics of cold-induced gene expression appeared accelerated and reduced in amplitude in Col-0 roots compared to rosette leaves ${ }^{8}$. In addition, expression of ribonucleoprotein (RNP) complexes (GO:0030529) and RNP biogenesis (GO:0022613) was activated in the cold. Expression of small nuclear RNP complexes (GO:0030532) that take part in splicing processes was constitutively activated in both mutants (Fig. 6C). Finally, five large biosynthesis and cytosol related GOs that contain ribosome and translation 
A

\begin{tabular}{|c|c|c|c|c|c|c|c|c|c|c|c|}
\hline \multirow[t]{2}{*}{ GO (Onthology) } & \multirow[t]{2}{*}{ Description } & \multirow[t]{2}{*}{ Genes } & \multicolumn{3}{|c|}{ Col-0 } & \multicolumn{3}{|c|}{ reil1-1 reil2-1 } & \multicolumn{3}{|c|}{ reil1-1 reil2-2 } \\
\hline & & & $\mathbf{0 d}$ & $1 \mathrm{~d}$ & $1 \mathrm{w}$ & $\mathbf{0 d}$ & $1 \mathrm{~d}$ & $1 \mathrm{w}$ & $0 \mathrm{~d}$ & $1 \mathrm{~d}$ & $1 \mathrm{w}$ \\
\hline GO:0009631 & $P$ cold acclimation & 22 & & $0.43 *$ & $0.70 *$ & -0.04 & 0.20 & $0.67 *$ & -0.25 & $0.46 *$ & $0.43 *$ \\
\hline GO:0009409 & $\mathrm{P}$ response to cold & 263 & & $0.08 *$ & $0.12 *$ & -0.03 & 0.05 & $0.10 *$ & 0.03 & $0.13 *$ & 0.05 \\
\hline GO:0009266 & $\mathrm{P}$ response to temperature stimulus & 395 & & -0.04 & -0.04 & 0.02 & -0.06 & $-0.10 *$ & $0.09 *$ & -0.03 & $-0.14 *$ \\
\hline GO:0010286 & $\mathrm{P}$ heat acclimation & 16 & & -0.10 & -0.02 & 0.26 & -0.04 & -0.03 & 0.06 & -0.17 & -0.19 \\
\hline GO:0009408 & $\mathrm{P}$ response to heat & 138 & & $-0.26 *$ & $-0.38 *$ & $0.12 *$ & $-0.24 *$ & $-0.48 *$ & $0.20 *$ & $-0.32 *$ & $-0.52 *$ \\
\hline GO:0009644 & $\mathrm{P}$ response to high light intensity & 47 & & $-0.31 *$ & $-0.21 *$ & $0.31 *$ & $-0.47 *$ & $-0.44 *$ & $0.37 *$ & $-0.41 *$ & $-0.48 *$ \\
\hline GO:0042542 & $\mathrm{P}$ response to hydrogen peroxide & 43 & & $-0.38 *$ & $-0.35 *$ & $0.27 *$ & $-0.45 *$ & $-0.53 *$ & $0.29 *$ & $-0.50 *$ & $-0.56 *$ \\
\hline GO:0000302 & $\mathrm{P}$ response to reactive oxygen species & 69 & & $-0.26 *$ & $-0.22 *$ & $0.22 *$ & $-0.30 *$ & $-0.33 *$ & $0.24 *$ & $-0.32 *$ & $-0.34 *$ \\
\hline
\end{tabular}

B

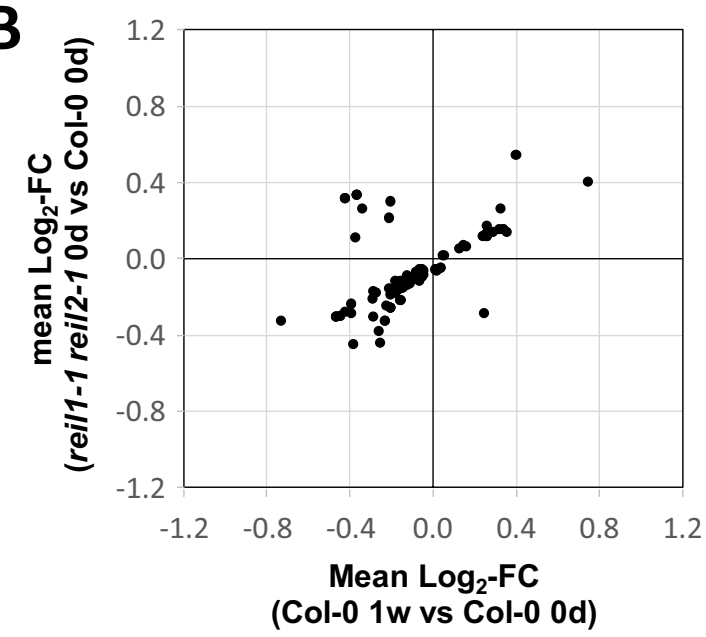

D

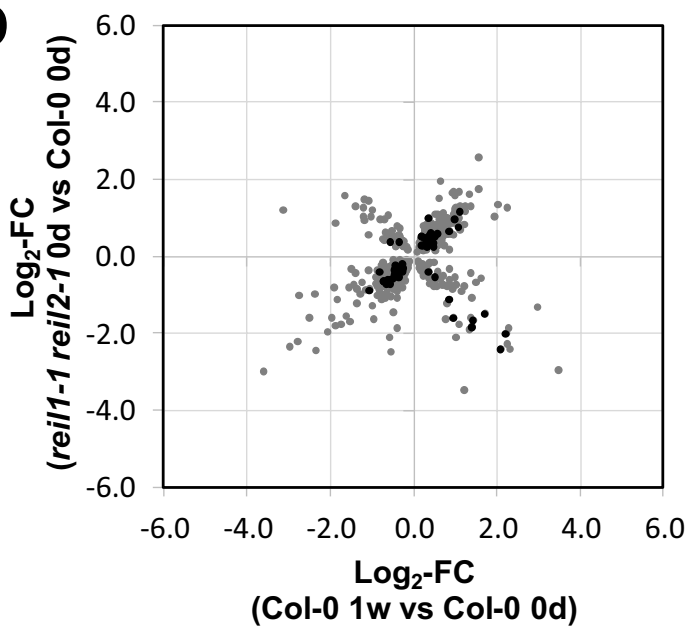

C

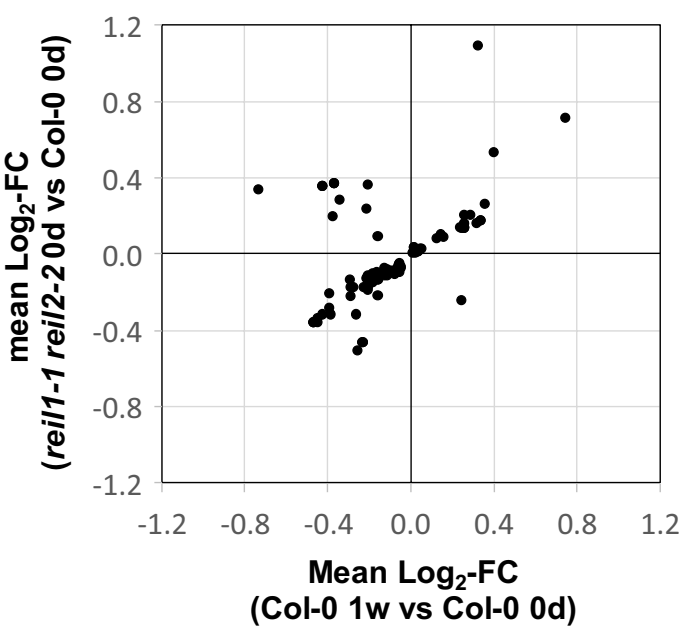

$\mathbf{E}$

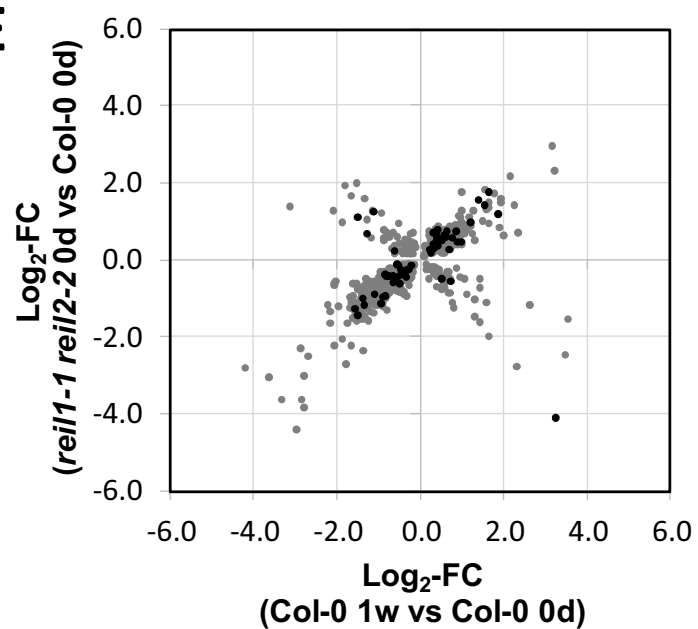

Figure 5. Functional enrichment and transcript correlation analyses of differential gene expression in the roots of Col- 0 , and the reil1-1 reil2-1 and reil1-1 reil2-2 double mutants in the non-acclimated state $\left(0\right.$ day, $\left.20^{\circ} \mathrm{C}\right)$ and shifted to $10^{\circ} \mathrm{C}$ cold for 1 day or 7 days. Differential gene expression is determined relative to non-acclimated Col- 0 at optimized temperature $20^{\circ} \mathrm{C}$. $(\mathbf{A}) \mathrm{Mean}$ $\log _{2}$-fold changes (FC) of temperature related GO terms. Significant positive or negative functional enrichments, i.e. FDR-adjusted $P$-values $<0.05$, are indicated by asterisks. The heat map color scale ranges from $\log _{2}-\mathrm{FC}+1.5$ (red) to -1.5 (blue). Mean $\log _{2}$-FC, Z-scores, and FDR-adjusted $P$-values of gene sets from $2145 \mathrm{GO}$ terms are calculated by parametric analysis of gene set enrichment $(\mathrm{PAGE})^{19}$ (Supplemental Table S3). (B) Mean $\log _{2}$-FC of shared significantly enriched GO terms from the non-acclimated reil1-1 reil21 mutant $(0 \mathrm{~d})$ compared to acclimated Col-0 at 7 days after shift. (C) Mean $\log _{2}$-FC of shared significantly enriched GO terms from the non-acclimated reil1-1 reil2-2 mutant $(0 \mathrm{~d})$ compared to acclimated Col- 0 at 7 days after shift. Note that both double mutants and cold acclimated Col-0 shared 100 GO terms of 2145 (FDR-adjusted $P<0.05$ ). (D) Significantly changed transcripts of non-acclimated reil1-1 reil2-1 relative to non-acclimated Col-0 compared to significant cold-responsive transcripts of Col- 0 at 7 days after cold shift $(\mathrm{P}<0.05$, gray, $\mathrm{P}<0.01$ black, heteroscedastic T-tests). (E) Significantly changed transcripts of non-acclimated reil1-1 reil2-2 relative to non-acclimated Col- 0 compared to significant cold-responsive transcripts of Col- 0 at 7 days after cold shift $(\mathrm{P}<0.05$, gray, $\mathrm{P}<0.01$ black, heteroscedastic $t$ tests). Note that correlation coefficients are not calculated because assumptions of linear correlation do not apply to the full set of observations. 


\begin{tabular}{|c|c|c|c|c|c|c|c|c|c|c|c|}
\hline \multirow[t]{2}{*}{ GO (Onthology } & \multirow[t]{2}{*}{ Description } & \multirow[t]{2}{*}{ Genes } & \multicolumn{3}{|c|}{ Col-0 } & \multicolumn{3}{|c|}{ reil1-1 reil2-1 } & \multicolumn{3}{|c|}{ reil1-1 reil2-2 } \\
\hline & & & $\mathbf{0} \mathbf{d}$ & $1 \mathrm{~d}$ & $1 \mathrm{w}$ & $\mathbf{0 d}$ & $1 \mathrm{~d}$ & $1 \mathrm{w}$ & $\mathbf{0 d}$ & $1 \mathrm{~d}$ & $1 \mathrm{w}$ \\
\hline GO:0042254 & $\mathrm{P}$ ribosome biogenesis & 151 & & $0.27 *$ & 0.07 & $-0.12 *$ & $0.38 *$ & $0.15 *$ & $0.13 *$ & $0.33 *$ & $0.19 *$ \\
\hline GO:0005840 & C ribosome & 428 & & $0.20 *$ & -0.02 & $-0.14 *$ & $0.29 *$ & $0.10 *$ & $0.09 *$ & $0.23 *$ & $0.15 *$ \\
\hline GO:0022626 & C cytosolic ribosome & 277 & & $0.23 *$ & -0.04 & $-0.22 *$ & $0.37 *$ & $0.11 *$ & $0.11 *$ & $0.33 *$ & $0.20 *$ \\
\hline GO:0022625 & C cytosolic large ribosomal subunit & 133 & & $0.27 *$ & -0.01 & $-0.22 *$ & $0.42 *$ & $0.15 *$ & $0.13 *$ & $0.36 *$ & $0.23 *$ \\
\hline GO:0022627 & C cytosolic small ribosomal subunit & 109 & & $0.22 *$ & -0.06 & $-0.24 *$ & $0.37 *$ & $0.11 *$ & 0.07 & $0.32 *$ & $0.18 *$ \\
\hline GO:0000313 & C organellar ribosome & 42 & & 0.13 & 0.09 & 0.15 & 0.05 & 0.13 & 0.04 & -0.17 & 0.04 \\
\hline GO:0000315 & C organellar large ribosomal subunit & 20 & & 0.10 & 0.12 & 0.23 & 0.01 & 0.11 & 0.05 & -0.21 & 0.06 \\
\hline GO:0000314 & C organellar small ribosomal subunit & 21 & & 0.15 & 0.05 & 0.09 & 0.08 & 0.15 & 0.01 & -0.14 & 0.02 \\
\hline GO:0009547 & C plastid ribosome & 30 & & $0.20 *$ & 0.17 & $0.29 *$ & 0.12 & $0.23 *$ & 0.13 & -0.15 & 0.08 \\
\hline GO:0000311 & C plastid large ribosomal subunit & 16 & & 0.18 & 0.19 & $0.35 *$ & 0.11 & 0.19 & 0.18 & -0.13 & 0.10 \\
\hline GO:0000312 & C plastid small ribosomal subunit & 14 & & 0.22 & 0.14 & 0.23 & 0.13 & 0.28 & 0.08 & -0.17 & 0.05 \\
\hline GO:0005761 & C mitochondrial ribosome & 12 & & -0.04 & -0.09 & -0.20 & -0.11 & -0.12 & -0.19 & -0.21 & -0.05 \\
\hline
\end{tabular}

\begin{tabular}{|c|c|c|c|c|c|c|c|c|c|c|c|}
\hline GO (Onthology) & \multirow{2}{*}{ Description } & \multirow[t]{2}{*}{ Genes } & \multicolumn{3}{|c|}{ Col-0 } & \multicolumn{3}{|c|}{ reil1-1 reil2-1 } & \multicolumn{3}{|c|}{ reil1-1 reil2-2 } \\
\hline & & & $0 \mathrm{~d}$ & $1 \mathrm{~d}$ & $1 \mathrm{w}$ & $\mathbf{0 d}$ & $1 \mathrm{~d}$ & $1 \mathrm{w}$ & $\mathbf{0}$ d & $1 \mathrm{~d}$ & $1 \mathrm{w}$ \\
\hline GO:0006412 & $\mathrm{P}$ translation & 620 & & $0.19 *$ & 0.00 & $-0.07 *$ & $0.28 *$ & $0.10 *$ & $0.07 *$ & $0.19 *$ & $0.12 *$ \\
\hline GO:0008135 & F translation factor activity & 145 & & $0.15 *$ & 0.02 & 0.03 & $0.24 *$ & 0.08 & $0.09 *$ & $0.11 *$ & 0.02 \\
\hline GO:0003743 & F translation initiation factor (IF) activity & 95 & & $0.16 *$ & 0.02 & 0.04 & $0.24 *$ & 0.09 & 0.10 & $0.14 *$ & 0.04 \\
\hline GO:0005852 & C eukaryotic translation IF3 complex & 10 & & $0.33 *$ & 0.11 & 0.18 & $0.64 *$ & $0.56 *$ & 0.26 & $0.52 *$ & $0.46 *$ \\
\hline GO:0003746 & F translation elongation factor activity & 33 & & $0.19 *$ & 0.02 & -0.06 & $0.24 *$ & 0.01 & 0.07 & 0.09 & -0.01 \\
\hline GO:0003747 & F translation release factor activity & 13 & & 0.01 & 0.06 & 0.11 & 0.01 & 0.06 & 0.05 & -0.17 & -0.08 \\
\hline GO:0006418 & P tRNA aminoacylation for translation & 69 & & $0.14 *$ & 0.03 & 0.04 & $0.20 *$ & 0.00 & -0.01 & 0.04 & 0.03 \\
\hline
\end{tabular}

\begin{tabular}{|c|c|c|c|c|c|c|c|c|c|c|c|}
\hline GO (Onthology) & \multirow[t]{2}{*}{ Description } & \multirow[t]{2}{*}{ Genes } & \multicolumn{3}{|c|}{ Col-0 } & \multicolumn{3}{|c|}{ reil1-1 reil2-1 } & \multicolumn{3}{|c|}{ reil1-1 reil2-2 } \\
\hline & & & $0 \mathrm{~d}$ & $1 \mathrm{~d}$ & $1 \mathrm{w}$ & od & $1 \mathrm{~d}$ & $1 \mathrm{w}$ & Od & $1 \mathrm{~d}$ & $1 \mathrm{w}$ \\
\hline GO:0030529 & C ribonucleoprotein (RNP) complex & 514 & & $0.21 *$ & 0.02 & $-0.10 *$ & $0.28 *$ & $0.12 *$ & $0.11 *$ & $0.24 *$ & $0.16 *$ \\
\hline GO:0022613 & P RNP complex biogenesis & 159 & & $0.26 *$ & 0.06 & $-0.12 *$ & $0.36 *$ & $0.13 *$ & $0.13 *$ & $0.30 *$ & $0.17 *$ \\
\hline GO:0022618 & P RNP complex assembly & 12 & & 0.07 & -0.08 & -0.10 & 0.09 & 0.02 & 0.12 & -0.06 & -0.11 \\
\hline GO:0005732 & C small nucleolar RNP complex & 30 & & $0.26 *$ & 0.16 & -0.09 & $0.28 *$ & 0.17 & 0.14 & $0.33 *$ & $0.21 *$ \\
\hline GO:0030532 & C small nuclear RNP complex & 13 & & $0.65 *$ & $0.74 *$ & $0.41 *$ & $0.53 *$ & $0.87 *$ & $0.72 *$ & $0.90 *$ & $0.83 *$ \\
\hline
\end{tabular}

Figure 6. Functional enrichment analyses of differential gene expression in roots of Col-0, and the reill-1 reil2-1 and reil1-1 reil2-2 double mutants in the non-acclimated state $\left(0\right.$ day, $\left.20^{\circ} \mathrm{C}\right)$ and shifted to $10{ }^{\circ} \mathrm{C}$ cold for 1 day or 7 days. Differential gene expression is determined relative to non-acclimated Col-0 at optimized temperature $20^{\circ} \mathrm{C}$. (A) Mean $\log _{2}$-fold changes (FC) of selected ribosome biogenesis and ribosome related GO terms. Note the prolonged and stronger activation of cytosolic ribosome related genes in the mutants. (B) Mean $\log _{2}$-FCs of selected translation related GO terms. (C) Mean $\log _{2}$-FCs of selected ribonucleoprotein related GO terms. ( $\mathrm{C}=$ cellular component, $\mathrm{P}=$ biological process, $\mathrm{F}=$ molecular function). Significant positive or negative functional enrichments, i.e. FDR-adjusted $P$-values $<0.05$, are indicated by asterisks. The heat map color scale ranges from $\log _{2}-\mathrm{FC}+1.5$ (red) to -1.5 (blue). Mean $\log _{2}-\mathrm{FC}, \mathrm{z}$-scores, and FDR-adjusted $P$-values of gene sets from $2145 \mathrm{GO}$ terms are calculated by parametric analysis of gene set enrichment (PAGE) ${ }^{19}$ (Supplemental Table S3).

related genes responded in a pattern that was similar to cytosolic ribosomes (Supplemental Fig. S6B, Supplemental Table S3).

We added a more detailed study of RP gene expression from the 40S SSU (104 transcripts covered by our current study) and the 60S LSU (159 transcripts) complexes. We compared expression of cytosolic RPs to the plastid 30S (30 transcripts) and 50S (41 transcripts) RPs. This comparison revealed in part opposing effects of reil deficiency on structural cytosolic and plastid RPs (Fig. 7). At $10^{\circ} \mathrm{C}$, expression of $40 \mathrm{~S}$ and $60 \mathrm{~S}$ RPs coordinately increased in both mutants relative to Col-0 at each time point. In the non-acclimated state, reill-1 reil21 roots reduced cytosolic RP expression whereas reil1-1 reil2-2 roots had increased cytosolic RP transcripts (Fig. 7A). Expression of plastid RPs inversely increased in the non-acclimated state and decreased after shift to cold (Fig. 7A).

Analysis of cytosolic RP transcripts by pairwise comparisons at each time point and by two factorial analyses of variance (ANOVA) that tested for the influence of the factors, mutant genotype and time of cold exposure, indicated heterogeneity of RP gene expression changes at single gene levels (Fig. 7B, Supplemental Table S2). Many RP gene families comprised members that were either significantly affected by the factor cold or the genotype. We also observed RP paralogs with significant interactions between genotype and cold exposure or without significant changes (Fig. 7B).

In summary, reil deficiency globally activates cytosolic RP expression in roots with inverse effects on plastid RP gene expression. Reil deficiency can affect gene expression of paralogs from one family synergistically, e.g. RPL4A and RPL4D (Fig. 7B). Alternately, gene expression of RP paralogs from 40 and 60 S RP families can respond differentially, for example paralogs of the RPL3, RPP2 or RPP3, and of the RPS 23 , and RPS 27 gene 
A 405

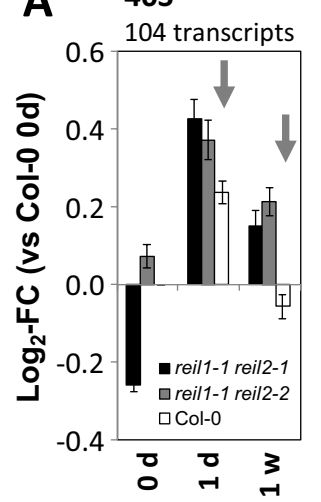

B 0.4

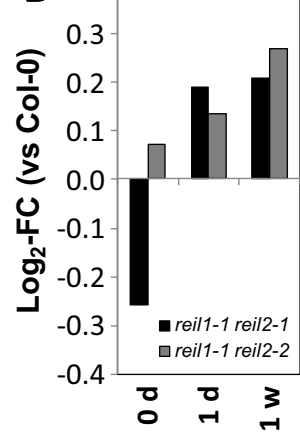

605
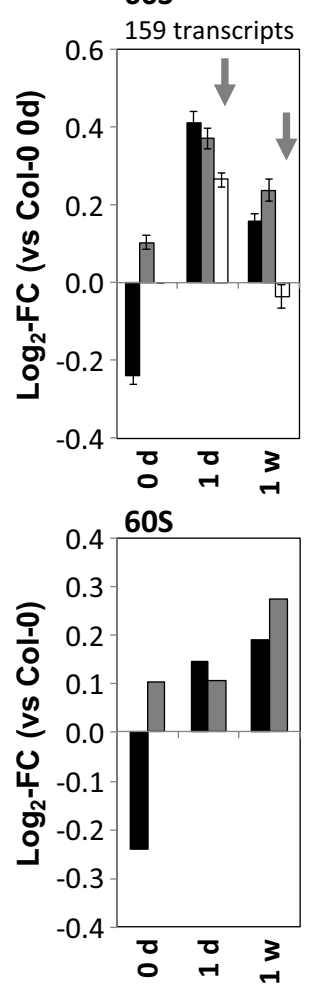

305
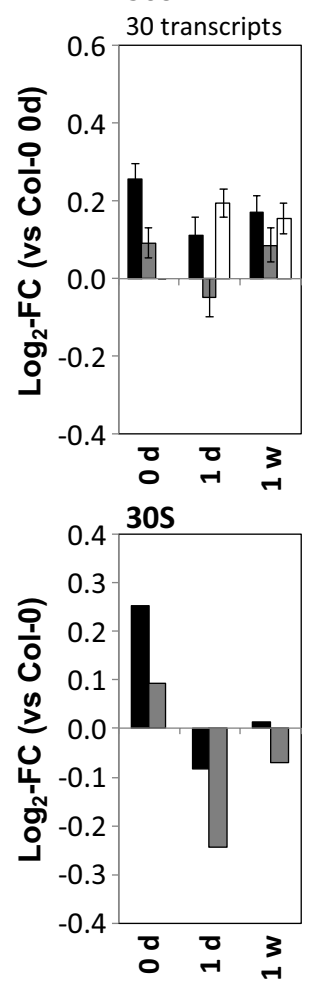

$50 S$
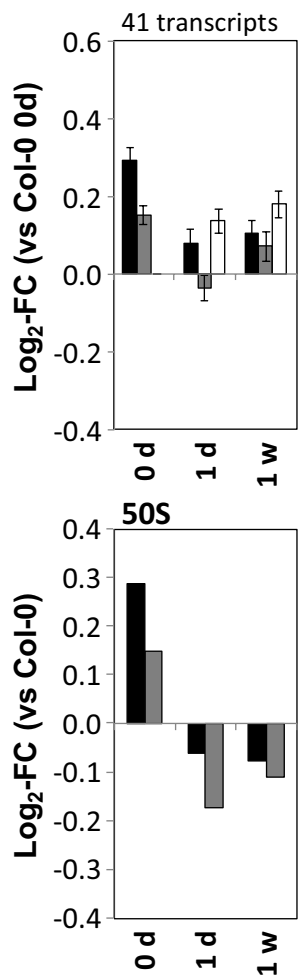

C

$\begin{array}{cc}\log _{2}-\mathrm{FC} & \log _{2}-\mathrm{FC} \\ (\text { reil1-1 reli2-1/Col-0) } & (\text { reil1-1 reil2-2/Col-0) }\end{array}$

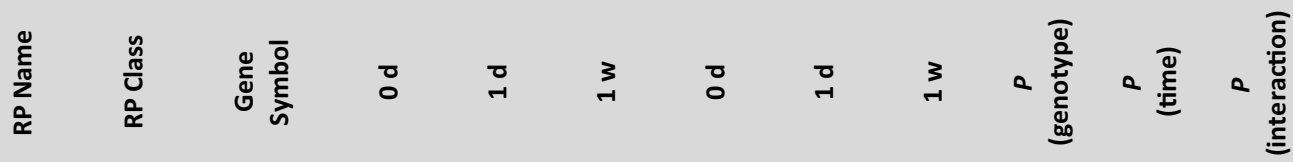

6OS LSU

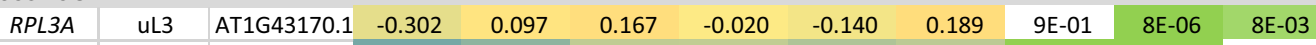

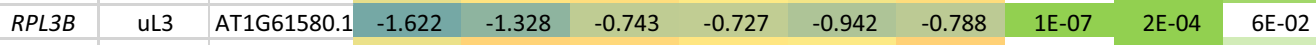

\begin{tabular}{l|l|l|llllllllll}
$R P L 4 A$ & UL4 & AT3G09630.1 & -0.224 & 0.316 & 0.247 & 0.073 & -0.029 & 0.332 & $3 \mathrm{E}-01$ & $9 \mathrm{E}-06$ & $3 \mathrm{E}-02$
\end{tabular}

\begin{tabular}{|l|l|l|l|l|l|l|l|l|l|l|l|}
$R P L 4 D$ & UL4 & AT5G02870.1 & -0.315 & 0.248 & 0.394 & -0.084 & 0.091 & 0.497 & $5 \mathrm{E}-02$ & $6 \mathrm{E}-05$ & $1 \mathrm{E}-03$
\end{tabular}

\begin{tabular}{l|l|llllllllll}
$R P P 2 A$ & $\mathrm{P} 1 / \mathrm{P} 2$ & $\mathrm{AT} 2 \mathrm{G} 27720.1$ & -0.196 & 0.009 & -0.001 & 0.040 & 0.050 & 0.121 & $4 \mathrm{E}-01$ & $2 \mathrm{E}-04$ & $9 \mathrm{E}-01$
\end{tabular}

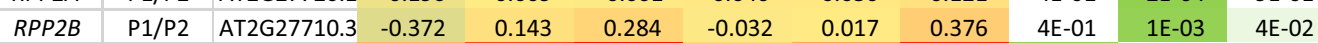

\begin{tabular}{|l|l|l|l|l|l|l|l|l|l|l|l|}
\hline$R P P 2 C$ & P1/P2 & AT3G28500.1 & 0.745 & 2.104 & 2.720 & 0.269 & 0.899 & 2.590 & $1 \mathrm{E}-03$ & $6 \mathrm{E}-01$ & $2 \mathrm{E}-01$ \\
\hline
\end{tabular}

\begin{tabular}{|l|l|l|l|l|l|llllll}
$R P P 2 D$ & P1/P2 & AT3G44590.1 & -0.289 & 0.064 & 0.268 & 0.205 & 0.169 & 0.323 & $1 \mathrm{E}-01$ & $1 \mathrm{E}-01$ & $4 \mathrm{E}-01$
\end{tabular}

\begin{tabular}{|l|l|l|l|l|l|l|l|l|l|l|l|l|}
\hline$R P P 2 E$ & P1/P2 & AT5G40040.1 & -2.382 & 0.315 & 2.035 & -0.603 & 0.200 & 2.420 & $5 \mathrm{E}-02$ & $2 \mathrm{E}-02$ & $1 \mathrm{E}-04$ \\
\hline
\end{tabular}

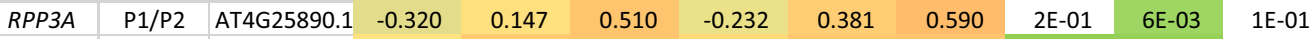

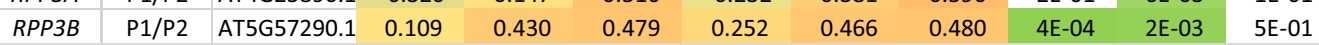

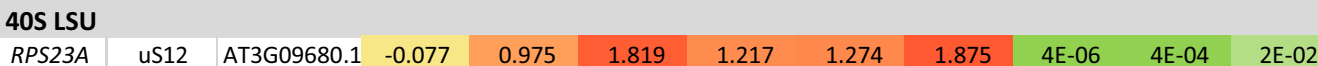

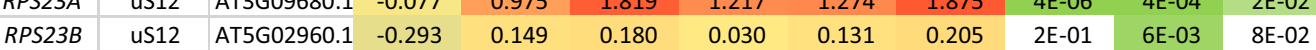

\begin{tabular}{|l|l|l|l|l|l|l|l|l|l|l|l|}
\hline RPS27A & eS27 & AT2G45710.1 & -0.208 & 0.510 & 0.268 & 0.072 & 0.675 & 0.459 & $8 \mathrm{E}-04$ & $5 \mathrm{E}-06$ & $3 \mathrm{E}-02$ \\
\hline
\end{tabular}

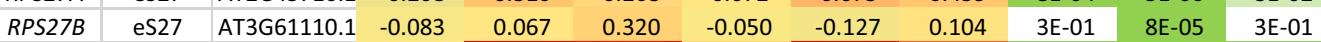

\begin{tabular}{|l|l|l|l|l|l|l|l|l|l|l|l}
$R P S 27 C$ & eS27 & AT3G61111.1 & 0.001 & 4.179 & 2.839 & 0.257 & 4.034 & 1.859 & $4 \mathrm{E}-09$ & $8 \mathrm{E}-10$ & $2 \mathrm{E}-06$
\end{tabular}

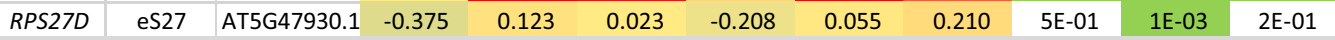

Figure 7. Differential expression of cytosolic and plastid ribosomal genes in reil1-1 reil2-1 and reil1-1 reil2-2 double mutant roots in the non-acclimated state $\left(0\right.$ day, $\left.20^{\circ} \mathrm{C}\right)$ and shifted to $10{ }^{\circ} \mathrm{C}$ cold for 1 day or 7 days. (A) Average $\log _{2}$-fold change (FC, means $+/$ - standard error) relative to non-acclimated Col-0 ( 0 day) of transcripts coding for ribosome proteins (RPs) from the cytosolic $40 \mathrm{~S}$ and $60 \mathrm{~S}$ subunits and from the plastid $30 \mathrm{~S}$ and $50 \mathrm{~S}$ subunits. Grey arrows indicate transcript accumulation of the mutants in the cold. (B) Average $\log _{2}$-fold changes relative to Col-0 at each time point calculated from the means of the data from (A). (C) $\log _{2}$-fold changes relative to Col-0 at each time point of selected cytosolic RP families. Two-factorial analysis of variance (ANOVA) indicates differential effects of the genotype, cold exposure (time) or the interaction of both on the expression of paralogous RPs from the $60 \mathrm{~S}$ and the $40 \mathrm{~S}$ subunits in the mutants. The three-color scale of the $\log _{2}$-FC heat map ranges from -3.0 (blue) to 0.0 (yellow) to $\geq+3.0$ (red). The two-color significance scale ranges from $P \leq 1.0 \times 10^{-10}$ (1E-10, dark green) to $P<0.05$ (5E-2, light green), $P \geq 0.05$ (white). 
families (Fig. 7B). We conclude that deregulation of RP gene or paralog transcription in the mutants may contribute to the accumulation delay of cytosolic ribosome in the cold. This defect, however, does not affect the non-acclimated state.

Constitutive transcriptional compensation responses to REIL deficiency indicate interaction of cytosolic ribosome maturation with nucleolar rRNA biogenesis and translation initiation. Several observations from the GO enrichment analysis indicated constitutive transcriptional changes before and after cold acclimation, e.g. related to the eIF3 complex (Fig. 6B). A detailed analysis of the ten members of GO:0005852 and the complete set of 13 subunits and in total 21 paralog genes constituting the plant eIF3 complex ${ }^{20,21}$ revealed a single gene, namely eIF3C-2 (AT3G22860), that was highly and constitutively activated (Fig. 8). This activation was in contrast to its paralog, eIF3C-1 (AT3G56150), and other eIF3 components, such as eIF3G-2 (AT5G06000), that mostly mirrored cytosolic RP expression (Fig. 8). Applying the stringent criterion of significant constitutive changes relative to Col- 0 in both mutants, at all non-acclimated and cold acclimating states, with $P<0.05$ of each pairwise comparison (heteroscedastic T-tests), we discovered five additional constitutively accumulated transcripts. Next to two only marginally characterized F-box proteins (AT1G64540 and AT3G44120) and a MATH domain protein (AT3G29580), PISTILLATA (AT5G20240) and NUCLEOLIN 2 (NUC2, AT3G18610) matched our selection criteria. PISTILLATA is a floral homeotic protein, and transcription factor involved in the control of petal and stamen development. PISTILLATA is highly expressed in and near the root quiescent centers ${ }^{22,23}$. Apparently, PISTILLATA serves a yet elusive function in root development. Contrary to this enigmatic finding, function of NUC2 and of its paralog NUC1 provide a link to ribosome biogenesis ${ }^{24}$. NUC1 and NUC2 nucleolins have antagonistic roles in rRNA gene expression. Both are required for plant growth ${ }^{25}$. Similar to eIF3C2 and eIF3G2, the reil double mutants constitutively activate NUC2 expression whereas NUC1 expression mirrored cytosolic RP gene expression. Contrary to NUC1, NUC2 maintains or induces a repressive state of rDNA chromatin ${ }^{24}$.

In a meta-analysis, we compared constitutively changed root gene expression to non-acclimated and cold acclimating reil1-1 reil2-1 rosette leaves at 1 day, 7 days, and 3 weeks after cold shift ${ }^{8}$, see Supplemental Table S2 of $^{8}$. Transcripts of eIF3C2 (AT3G22860) and NUC2 (AT3G18610) are constitutively and significantly increased in reil1-1 reil2-1 mutant leaves with $P<0.05$ (heteroscedastic T-tests) of each pairwise comparison to Col-0. Transcripts of the F-box proteins, AT1G64540 and AT3G44120, increase significantly using the same significance threshold in mutant leaves at two or three of the compared time points, respectively. PISTILLATA and the MATH domain protein do not significantly change gene expression in reil1-1 reil2-1 mutant leaves.

Proteome analysis of ribosome preparations from Arabidopsis root tissue. For a qualitative comparative proteome analysis of ribosome complexes, we selected Col-0 wild type and the two double mutants, reil1-1 reil2-1 and reil1-1 reil2-2. Due to the limitations of the complex procedure ${ }^{8,10}$, we performed two independent experiments that each compared a pool of whole root systems from Col-0 wild type to a pool of one of the two double mutants. In the following, the two experiments are named DS1 (reil1-1 reil2-2) and DS2 (reil1-1 reil2-1). We analyzed the non-acclimated ribo-proteomes at day 0 to preparations obtained 7 days after shift to $10^{\circ} \mathrm{C}$ and sampled up to five non-translating ribosome complexes and the low-oligomer polysome fraction from sucrose density gradients. In this study, we avoided the period of reduced non-translating subunit abundance of the mutants at day 1 after cold shift and focused on the recovering ribo-proteome (Figs. 1, 2).

We first analyzed the RP composition of each fraction to identify and align the ribosome fractions between experiments that may differ slightly in position, but not in sequence of ribosome complexes within the sucrose gradients. For alignment of fractions, we used the sums of abundances of all detected 40S and 60S RPs, of the plastid and mitochondrial $30 \mathrm{~S}$ and 50S RPs, as well as the abundances of Arabidopsis EUKARYOTIC TRANSLATION INITIATION FACTOR 6, eIF6A (AT3G55620), and NONSENSE-MEDIATED mRNA DECAY 3 protein, NMD3 (AT2G03820) (Supplemental Table S4). eIF6A and NMD3 are homologs of the yeast cytosolic 60S maturation factors, TIF $6^{26}$ and NMD ${ }^{27}$. NMD3 and TIF6 are bound to translationally inactive pre-60S ribosome complexes $^{2,28}$ and in the case of TIF6 also indicate the position of NOP7-affinity purified 66S pre-ribosomes ${ }^{29}$. Next to the polysome fraction, we obtained from both experiments a fraction enriched in $40 \mathrm{~S}$ and organelle $30 \mathrm{~S}$ RPs, designated the 30S/40S fraction, a fraction of organelle 50S RPs (50S), a fraction that contained predominantly $60 \mathrm{~S}$ RPs $(60 \mathrm{~S})$ and a mixed fraction of $80 \mathrm{~S}$ and $60 \mathrm{~S}$ complexes (60S/80S) (Supplemental Fig. S7). In our current experiment, we did not reproducibly obtain proteome profiles of $80 \mathrm{~S}$ fractions. For this reason, and because the $80 \mathrm{~S}$ fraction is a difficult to interpret mixture of $\mathrm{KCl}$-sensitive, non-translating and non $\mathrm{KCl}$-sensitive, translating subpopulations (Fig. 4), we omitted $80 \mathrm{~S}$ fractions from the current analyses. Both, the $60 \mathrm{~S}$ and the $60 \mathrm{~S} / 80 \mathrm{~S}$ fractions of experiments DS1 and DS2 contained eIF6A and NMD3 indicative of plant equivalents of $60 \mathrm{~S}$ and $66 \mathrm{~S}$ pre-ribosome complexes. In DS1, REIL proteins were detected only by few peptides and a sequence coverage of $<8.0 \%$. Therefore, we did not analyze the abundance of REIL proteins in DS1. In DS2, however, the $60 \mathrm{~S}$ and $60 \mathrm{~S} / 80 \mathrm{~S}$ fractions of the Col-0 wild type contained the REIL2 protein. Identification of REIL2 in DS2 was supported by up to six peptides and $22.3 \%$ sequence coverage.

The variation of cytosolic, plastid, and mitochondrial RP abundances among the four sample pools and resulting fractions of our proteomics experiments (Supplemental Fig. S7) prompted us to investigate total RP abundances summed up across all ribosome fractions of each of the samples (Supplemental Table S5). The reil1.1 reil2.1 sample of DS2 fully recovered after 7 days in the cold in terms of total 40S and 60S abundances. At the same time after cold shift, the reill.1 reil2.2 sample of DS1 still lacked in total 40S and 60S abundance. This observation was in agreement with our previous spectrophotometric analysis of the sedimentation profiles at seven days after cold shift (Fig. 2D). The two mutant samples also differed in organelle RPs. Reil1.1 reil2.1 roots 

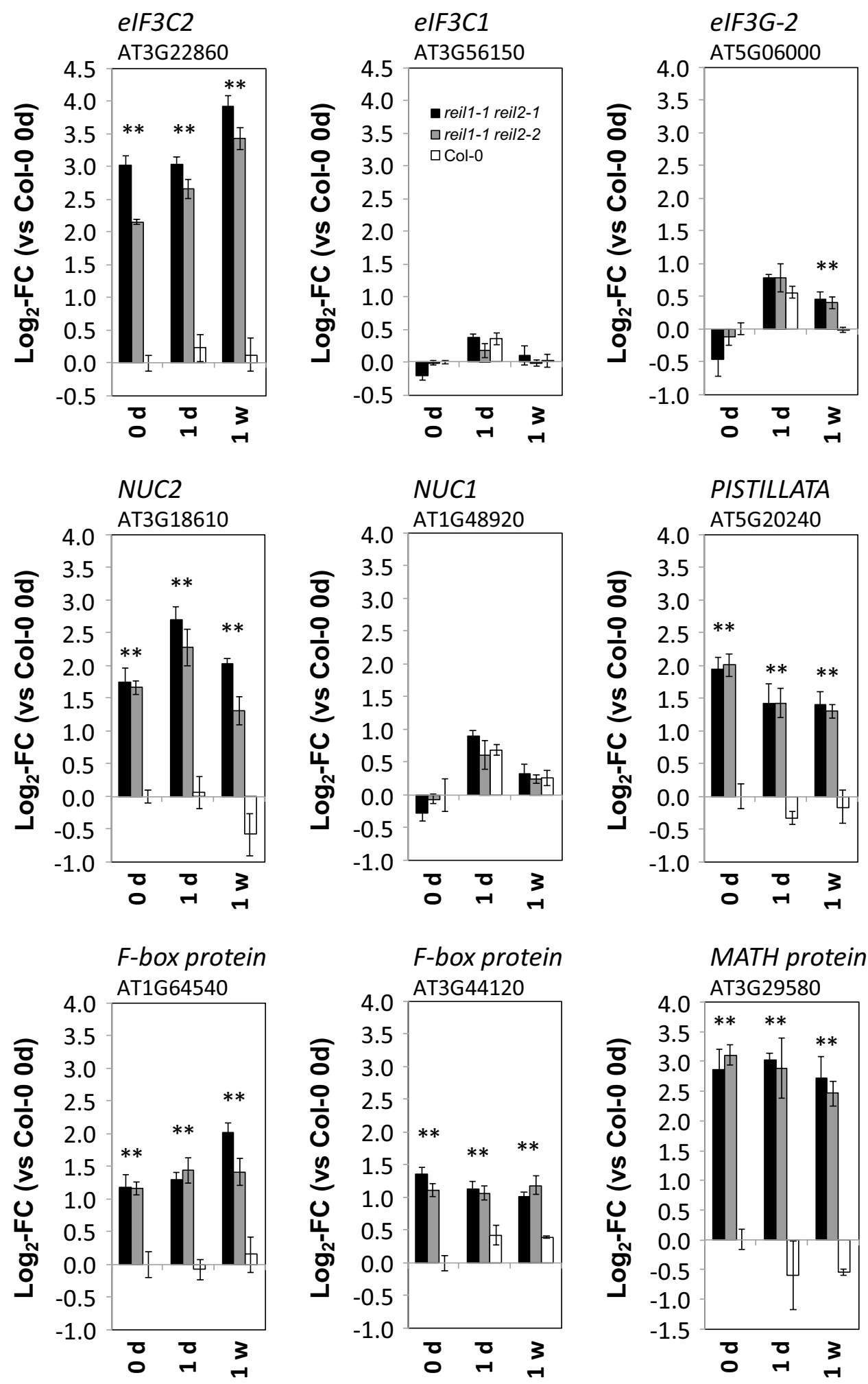

Figure 8. Differential expression of six genes that accumulate constitutively in reil1-1 reil2-1 and reil1-1 reil2-2 double mutant roots (means $+/$ - standard error, $\mathrm{n}=3$ ). eIF3C2, eIF3C1 and eIF3G2 code for paralogs of the eukaryotic translation initiation factor 3 multi-protein complex. NUC1 and NUC2 are plant nucleolins. Asterisks indicate $P<0.05$ (heteroscedastic Student's t-test) of comparisons to Col-0 at the same time point. NUC1, eIF3C1 and eIF3G2 are added to this figure to demonstrate the specific mutant effect on paralog transcription. 
decreased organelle RPs especially plastid and mitochondrial 50S RPs, both before and after cold shift. Inversely, reil1.1 reil2.2 accumulated 50S RPs (Supplemental Table S5).

For our subsequent datamining, we focused on common responses of both mutants and searched for proteins that consistently accumulated or decreased relative to Col- 0 wild type. Given the cold sensitivity of the reil mutants we first looked for common changes of the two reil double mutants relative to Col- 0 wild type in the cold. Subsequently extracted the subset of common changes in the mutants that were also present prior to the cold shift at $20^{\circ} \mathrm{C}$. We took into account the obvious variation $40 \mathrm{~S}$ SSU and $60 \mathrm{~S}$ LSU abundances among the ribosome fractions and among the four analyzed sample pools and applied a normalization procedure. To normalize the $60 \mathrm{~S}$ and $60 / 80 \mathrm{~S}$ fractions, we divided the abundance of the single observed proteins in each fraction to the abundance sum of all $60 \mathrm{~S}$ RPs from the respective fraction. The $30 \mathrm{~S} / 40 \mathrm{~S}$ fractions were normalized separately by the sum of all detected 40S RPs. The normalization enabled analyses of compositional changes of cytosolic RPs and co-purified non-ribosomal proteins in the non-translating SSU and LSU fractions. We analyzed increases and decreases in the mutants relative to the Col-0 wild type by $\log _{2}$-fold changes $\left(\log _{2}\right.$-FC) (Figs. 9, 10, 11). The presence of a protein in a mutant sample and absence in corresponding Col- 0 wild type classified as accumulation $(+)$, absence in the mutant and presence in Col-0 classified as decrease (-).

The experiments DS1 and DS2 differed slightly in sensitivity and corresponding complexity of monitored RPs. DS1 yielded a more complex data set with 2820 compared to 1675 detected proteins in DS2 (Supplemental Table S5). The number of detected cytosolic RPs and RP paralogs was, however, almost equal, 175 in DS1 and 174 in DS2 (Supplemental Table S6). The experiments shared 162 cytosolic RPs and RP paralogs of in total 1485 common proteins. The detection of cytosolic RPs was mostly paralog specific or represented RP families with identical amino acid sequences. Twenty-six (DS1) and 21 (DS2) cytosolic RPs lacked paralog specificity (Supplemental Table S6). Our analysis did not differentiate splice variants. In agreement with the cold-sensitivity of the mutants, we found more shared changes of normalized protein abundances in the cold than prior to cold shift. Most of the observed changes were larger in magnitude in DS1 (reil1.1 reil2.2) than in DS2 (reil1.1 reil2.1). The current experiments, however, do not allow interpretation of these observations either as a difference between the two mutants or as a result of changed kinetics after cold shift (Fig. 2).

60S RPs and RP paralogs differentially accumulate in non-translating 60S fractions of reil double mutants. We selected 61 proteins with shared increases ( 36 proteins) or decreases ( 25 proteins) relative to the sum of abundances of all detected $60 \mathrm{~S}$ RPs at $10^{\circ} \mathrm{C}$ in the non-translating $60 \mathrm{~S}$ fractions of reil double mutants (Supplemental Table S7A). Co-purified proteins with an abundance maximum in the 50S fraction were removed from this analysis. Most observed changes were below twofold. Eight proteins increased consistently at $10{ }^{\circ} \mathrm{C}$ and $20^{\circ} \mathrm{C}$, seven decreased. The remaining 46 proteins changed in part inversely at $20^{\circ} \mathrm{C}$ in both reil double mutants, were not detectable at optimized temperature, or differed at optimized temperature between mutants (Supplemental Table S7A).

The majority of the proteins were structural cytosolic RPs (45) that either increased (20) or decreased (25). RPL3A (AT1G43170), RPL10aB (AT2G27530), RPL12C (AT5G60670), and RPL30C (AT3G18740) increased consistently in the reil double mutants, RPL3B (AT1G61580), RPL7aB (AT3G62870), RPL13aD (AT5G48760), RPL24B (AT3G53020), RPL26A (AT3G49910), RPL37aC (AT3G60245) decreased (Fig. 9). Among the 61 changed proteins were cytosolic RPs that are assembled in the nucleus and a set of RPs that are added or exchanged in the cytosol after export from the nucleus, such as, already mentioned RPL24B (AT3G53020) of the eL24 family and RPL10A (AT1G14320) and RPL10C (AT3G11250) of the uL16 family, P-stalk proteins, RPP0B (AT3G09200) and RPP0C (AT3G11250) of the uL10 family or RPP1A (AT1G01100), RPP1B (AT4G00810), RPP1C (AT5G47700), RPP2D (AT3G44590) of the P1/P2 family (Fig. 9, Supplemental Figure S8, Supplemental Table S7A). RPP1A, RPP1B, RPP1C and RPP0B decreased at $10^{\circ} \mathrm{C}$ and increased at $20^{\circ} \mathrm{C}$ relative to wild type (Fig. 9, Supplemental Figure S8).

Homologs of yeast $66 \mathrm{~S}$ pre-ribosome constituents and $60 \mathrm{~S}$ biogenesis factors accumulate in non-translating 605 fractions of reil double mutants. The sixteen non-structural cytosolic RPs among the set of 61 differentially accumulating proteins were with two exceptions, namely a root tip expressed LEA protein (AT5G60530) and a R3H domain protein (AT1G03250), homologs of known yeast 60S biogenesis factors and $66 \mathrm{~S}$ pre-ribosome constituents (Fig. 10). We found the homologs of yeast TIF6, NMD3, RLP24, NOP7, NOG1, NOG2 (alias NUG2), RPF2, NSA2, MAK16, RSA4, CIC1 (alias NSA3), EBP2, HAS1 and YTM1. All accumulated in the non-translating $60 \mathrm{~S}$ fractions at $10^{\circ} \mathrm{C}$. The TIF6-, NMD3- and RLP24-homologs and the $\mathrm{R} 3 \mathrm{H}$ domain protein accumulated in addition at $20^{\circ} \mathrm{C}$. Next to yeast NOP7, the biogenesis factors, yeast TIF6, NOG1, MAK16, HAS1, EBP2, NSA2, CIC1, YTM1 and RLP24 are non-structural ribosomal proteins that are associated with $66 \mathrm{~S}$ pre-ribosomes, specifically a fraction that can be NOP7 affinity-purified ${ }^{29,30}$. The remaining NMD3, NOG2, RPF2, and RSA4 proteins are part of diverse other pre-60S ribosome complexes. In detail and again assuming basic homology of plant and yeast 60S biogenesis, NMD3 is a nuclear export adaptor of pre-60S subunits that is part of a pre-60S ribosome carrying TIF6, LSG1 and the yeast REI-homolog, REH1 ${ }^{31}$. Like TIF6, RLP24 or NOG1, NMD3 is released from pre-60S ribosomes in the final cytosolic maturation steps and recycled ${ }^{2,27}$. NOG2 (alias NUG2) precedes NMD3 binding to pre-60S ribosomes at overlapping binding sites prior to nuclear export ${ }^{32}$. RSA4 is present on NOG2 associated particles and is a co-substrate of the REA1 remodeling factor that is required for NOG2 release from pre-60S ribosomes ${ }^{32}$. Finally, NOG $2^{33}$ associates with pre-60S ribosomes already in the nucleolus and together with NSA2 is thought to be part of a 66SB pre-ribosome and required for $27 \mathrm{SB}$ pre-rRNA processing into $25 \mathrm{~S} \mathrm{rRNA}^{33}$. Similarly, RFP2 ${ }^{34}$ predominantly localizes to the nucleolus and associates with $66 \mathrm{~S}$ pre-ribosomes by an alternative recruiting pathway. RFP2 is also required for 27SB pre-rRNA processing ${ }^{33}$. HAS1 is a RNA helicase that takes part in both small and large ribosomal subunit 
60S LSU

\begin{tabular}{|c|c|c|c|c|c|c|}
\hline & & & & \\
\hline AT4G27090 & RPL14B & eL14 & 0.47 & 0.22 & -0.74 & -0.40 \\
\hline АТЗG14600 & RPL18aC & eL20 & -1.27 & -0.36 & 0.13 & 0.24 \\
\hline АТЗG53020 & RPL24B & eL24 & -0.54 & -1.04 & -0.64 & -1.25 \\
\hline АТ3G18740 & RPL30C & eL30 & 1.06 & 0.68 & 0.51 & 0.39 \\
\hline AT1G41880 & RPL35aB & eL33 & -0.21 & -0.76 & 0.66 & 1.40 \\
\hline АТ3G60245 & RPL37aC & eL43 & -1.46 & -0.32 & -0.58 & -0.40 \\
\hline АT3G62870 & RPL7aB & eL8 & -0.80 & -0.45 & -0.12 & -0.45 \\
\hline AT1G01100 & RPP1A & P1/P2 & -0.22 & -0.52 & 0.51 & 0.60 \\
\hline AT5G47700 & RPP1C & P1/P2 & -0.73 & -0.49 & 0.16 & 0.63 \\
\hline AT4G00810 & RPP1B & P1/P2 & -0.19 & -0.33 & + & 0.10 \\
\hline АT2G27530 & RPL10aB & uL1 & 0.26 & 0.60 & 0.13 & 0.13 \\
\hline AT1G08360 & RPL10aA & uL1 & 0.58 & 1.72 & -0.31 & -0.85 \\
\hline AT5G22440 & RPL10aC & uL1 & -0.30 & -0.81 & 0.15 & 0.06 \\
\hline АТЗG09200 & RPPOB & uL10 & -0.32 & -1.10 & 0.63 & 0.82 \\
\hline AT5G60670 & RPL12C & uL11 & 0.90 & 1.09 & 0.37 & 0.22 \\
\hline AT5G48760 & RPL13aD & uL13 & -0.30 & -0.78 & -0.37 & -0.15 \\
\hline AT3G25520 & RPL5A & uL18 & 0.32 & 1.09 & -0.03 & -0.22 \\
\hline AT5G67510 & RPL26B & uL24 & 0.47 & + & -1.43 & -0.71 \\
\hline АТ3G49910 & RPL26A & uL24 & -0.28 & -0.37 & -0.12 & -0.08 \\
\hline AT1G43170 & RPL3A & uL3 & 0.17 & 0.23 & 0.26 & 0.02 \\
\hline AT1G61580 & RPL3B & uL3 & - & -2.03 & -1.44 & -0.92 \\
\hline AT2G01250 & RPL7B & uL30 & -0.09 & -0.11 & 0.49 & 0.18 \\
\hline АT2G44120 & RPL7C & uL30 & -0.56 & -0.90 & 0.48 & 0.23 \\
\hline AT4G10450 & RPL9D & uL6 & -0.92 & -0.40 & 0.09 & 0.62 \\
\hline \multicolumn{7}{|l|}{ 40S SSU } \\
\hline AT5G61170 & RPS19C & eS19 & -1.34 & -0.12 & 0.70 & 0.36 \\
\hline АТЗG02080 & RPS19A & eS19 & 0.53 & 2.00 & 0.42 & 1.16 \\
\hline АT3G53890 & RPS21B & eS21 & -0.09 & -1.80 & -0.41 & -0.23 \\
\hline АТЗG04920 & RPS24A & eS24 & 0.52 & 0.55 & 0.24 & 0.49 \\
\hline АT2G21580 & RPS25B & eS25 & 0.50 & + & -0.30 & -1.03 \\
\hline AT4G39200 & RPS25E & eS25 & 0.56 & + & 0.07 & 0.34 \\
\hline AT5G03850 & RPS28B & eS28 & 0.62 & + & -0.92 & -0.41 \\
\hline AT5G02960 & RPS23B & uS12 & -0.15 & -0.31 & 0.88 & 1.08 \\
\hline AT4G09800 & RPS18C & uS13 & 0.32 & 0.71 & -0.04 & -0.15 \\
\hline AT1G04270 & RPS15A & uS19 & 0.48 & + & + & 0.26 \\
\hline AT1G72370 & RPSaA & uS2 & 0.03 & 1.78 & -0.90 & -0.38 \\
\hline
\end{tabular}

Figure 9. Changes of RP composition in the non-translating 60S LSU and 40S SSU fractions of the reil1 reil2 double mutants 7 days after shift to $10^{\circ} \mathrm{C}$ and prior to cold shift at $20^{\circ} \mathrm{C}$. This selection shows RPs with responses shared between reil1-1 reil2-1 (DS2) and reil1-1 reil2-2 (DS1) at $10^{\circ} \mathrm{C}$ and $20^{\circ}$. $\log _{2}$-fold changes between mutants and Col-0 wild type are calculated after normalization of RP LFQ-abundances by the abundance sums of all detected 60S RPs in the respective fraction and combined across the $60 \mathrm{~S}$ and $60 \mathrm{~S} / 80 \mathrm{~S}$ fractions (top) or by the abundance sums of all detected 40S RPs in the 30S/40S fractions (bottom). Presence relative to absence in Col- 0 and $\log _{2}$-fold increases $>1$ are color-coded red, increases $<1$ are coded light-red. Absence relative to presence in Col- 0 and $\log _{2}$-fold decreases $<-1$ are color-coded blue, decreases $>-1$ are coded light-blue.

biogenesis ${ }^{35}$. HAS1 is present in yeast 90 S pre-ribosomes and has multiple binding sites in the $18 \mathrm{~S}$ rRNA and the $5.8 \mathrm{~S}$ and $25 \mathrm{~S}$ rRNAs. HAS1 remains associated with pre-40S and pre-60S complexes ${ }^{35}$. We, however, detected the plant homolog of HAS1 only in non-translating $60 \mathrm{~S}$ fractions of the cold-exposed reil double mutants (Supplemental Table S7A).

40S RPs and eukaryotic translation initiation factors differentially accumulate in non-translating $40 \mathrm{~S}$ fractions of reil double mutants. In comparison to the moderate number of highly specific ribosome and ribosome associated proteins (RAPs) that differentially accumulated in non-translating 60S fractions, we detected 146 proteins with shared increases (135 proteins) or decreases (11 proteins) at $10{ }^{\circ} \mathrm{C}$ in the non-translating $40 \mathrm{~S}$ fractions of the reil double mutants (Supplemental Table S7B). Only 21 of the selected proteins were structural cytosolic RPs that either increased (12 RPs) or decreased (9 RPs) normalized to the sum of abundances of detected 40S RPs. The majority of selected proteins were co-purified, non-ribosomal proteins, e.g. 2 organelle $30 \mathrm{~S}$ RPs, 3 nitrilases, 4 heat shock proteins, 6 components of the proteasome, 10 components of ATP synthases and a multitude of enzymes. We concluded that these observations are likely not bound to the non-translating $40 \mathrm{~S}$ fraction and omitted this set from further analysis. 
A

\begin{tabular}{|c|c|c|c|c|c|c|}
\hline Gene code & Yeast homolog & Name & $\begin{array}{c}\log _{2} \text { (reil1.1 } \\
\text { reil2.1/Col-0) } \\
10^{\circ} \mathrm{C} \text { (DS2) }\end{array}$ & $\begin{array}{c}\log _{2}(\text { reil1.1 } \\
\text { reil2.2/Col-0) } \\
10^{\circ} \mathrm{C}(\mathrm{DS} 1)\end{array}$ & \begin{tabular}{|c|}
$\log _{2}($ reil1.1 \\
reil2.1/Col-0) \\
$20^{\circ} \mathrm{C}$ (DS2)
\end{tabular} & $\begin{array}{c}\log _{2}(\text { reil1.1 } \\
\text { reil2.2/Col-0) } \\
20^{\circ} \mathrm{C} \text { (DS1) }\end{array}$ \\
\hline AT2G24500 & REI1/REH1 & REIL2 & - & NA & - & NA \\
\hline AT2G03820 & NMD3 & NMD3 & 1.44 & 0.66 & 1.01 & 2.60 \\
\hline AT3G55620 & TIF6 & elF6A & 0.11 & 1.81 & 0.34 & 0.72 \\
\hline AT2G44860 & RLP24 & RPL24C & 2.51 & 3.91 & 3.11 & + \\
\hline AT5G15550 & YTM1 & ATPEP2 & + & 1.35 & NA & + \\
\hline AT3G58660 & CIC1 (alias NSA3) & - & 0.82 & 1.64 & NA & + \\
\hline AT3G23620 & RPF2 & ARPF2 & 0.43 & 1.81 & -1.02 & 3.56 \\
\hline AT5G06360 & NSA2 & - & 0.62 & 1.95 & -0.96 & + \\
\hline AT5G14520 & NOP7 & PES & 1.14 & 2.36 & - & + \\
\hline AT1G50920 & NOG1 & ATNOG1-1 & 1.93 & 2.43 & -0.72 & 3.81 \\
\hline AT1G52980 & NOG2 (alias NUG2) & ATNUG2 & 0.26 & 2.76 & NA & 3.51 \\
\hline AT5G52820 & RSA4 & ATNLE1 & + & 3.10 & NA & + \\
\hline AT1G23280 & MAK16 & MAK16-related & + & 4.06 & NA & + \\
\hline AT3G22660 & EBP2 & EBP2 & + & + & NA & + \\
\hline AT3G18600 & HAS1 & - & + & + & NA & NA \\
\hline AT1G03250 & - & R3H-protein & 2.55 & + & + & + \\
\hline AT5G60530 & - & LEA-protein & + & + & NA & NA \\
\hline
\end{tabular}
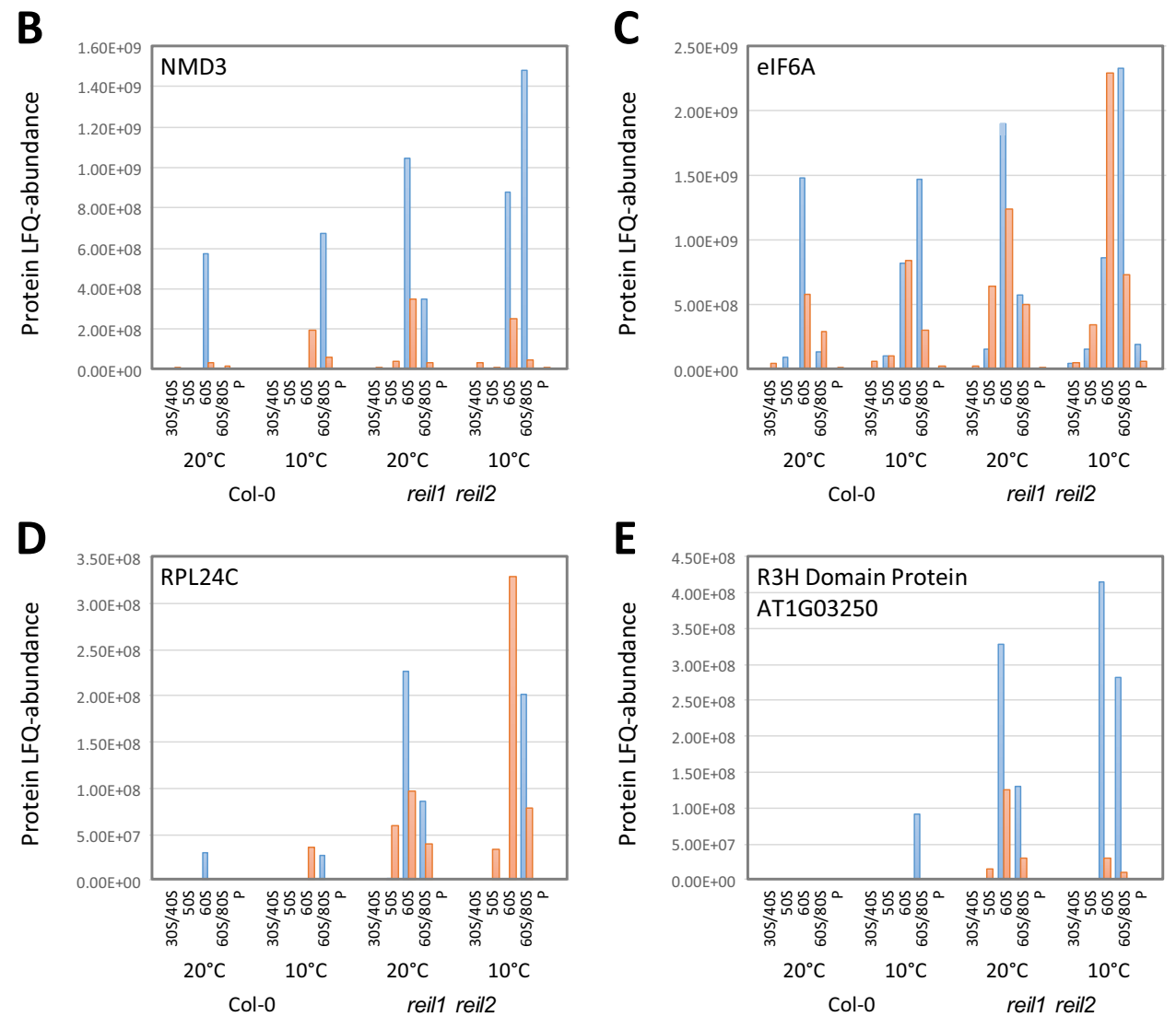

Figure 10. Changes of the composition of ribosome associated proteins in the non-translating 605 fractions of the reil1 reil2 double mutants 7 days after shift to $10^{\circ} \mathrm{C}$ and prior to cold shift at $20^{\circ} \mathrm{C}$. (A) REIL2 and the shared changes at $10^{\circ} \mathrm{C}$ between reil1-1 reil2-1 (DS2) and reil1-1 reil2-2 (DS1). Log $_{2}$-fold changes between mutants and Col- 0 wild type are calculated after normalization of protein LFQ-abundances by abundance sums of all detected 60S RPs and combined across the 60S and 60S/80S fractions. Presence in mutants relative to absence in Col- 0 and $\log _{2}$-fold increases $>1$ are color-coded red, increases $<1$ are light red. Absence relative to presence in Col- 0 and $\log _{2}$-fold decreases $<-1$ are color-coded blue, decreases $>-1$ are light-blue. Absence in both mutant and Col-0 is color-coded grey and indicated by NA (not available). (B-E) LFQ-abundance distributions of NMD3, eIF6A, RPL24C, and R3H domain Protein AT1G03250 across the sampled ribosome fractions and analyzed conditions (Experiment DS1, orange; experiment DS2, blue). Note the abundance maxima of the proteins in the non-translating $60 \mathrm{~S}$ and $60 / 80 \mathrm{~S}$ fractions. 
A

\begin{tabular}{|c|c|c|c|c|c|c|}
\hline Gene code & elF Name & elF Family & $\begin{array}{c}\log _{2} \text { (reil1.1 } \\
\text { reil2.1/Col-0) } \\
10^{\circ} \mathrm{C} \text { (DS2) }\end{array}$ & $\begin{array}{c}\log _{2}(\text { reil1.1 } \\
\text { reil2.2/Col-0) } \\
10^{\circ} \mathrm{C} \text { (DS1) }\end{array}$ & $\begin{array}{c}\log _{2}(\text { reil1.1 } \\
\text { reil2.1/Col-0) } \\
20^{\circ} \mathrm{C} \text { (DS2) }\end{array}$ & $\begin{array}{c}\log _{2} \text { (reil1.1 } \\
\text { reil2.2/Col-0) } \\
20^{\circ} \mathrm{C} \text { (DS1) }\end{array}$ \\
\hline AT4G11420 & elF3A1 & elF3a & 1.55 & + & -0.35 & 2.81 \\
\hline AT3G56150 & elF3C1/C2 & elF3c & 0.85 & + & -0.79 & + \\
\hline AT2G39990 & elF3F1 & elF3f & 0.22 & + & -0.82 & + \\
\hline
\end{tabular}

B

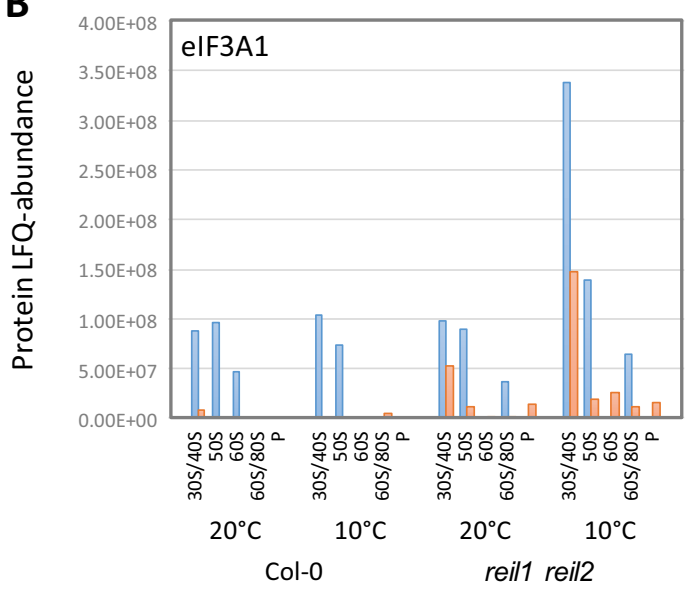

C

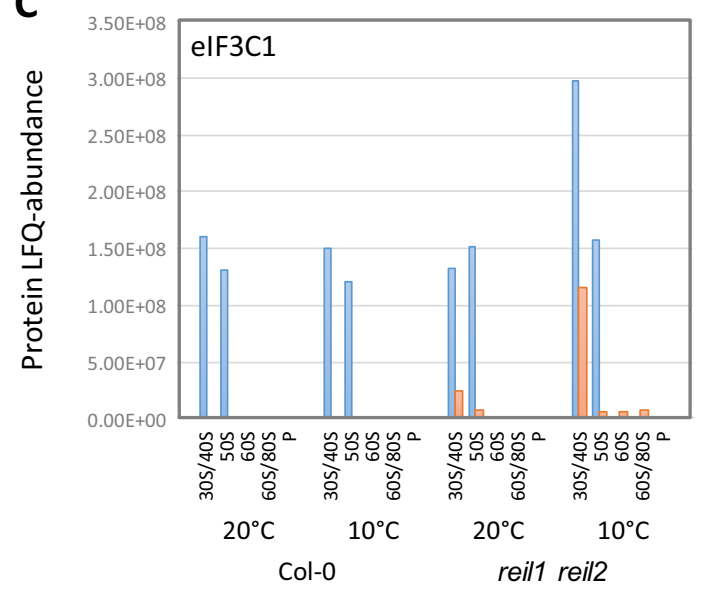

Figure 11. Changes of the composition of translation initiation factor 3 proteins in the non-translating $40 \mathrm{~S}$ fraction of the reil1 reil2 double mutants 7 days after shift to $10^{\circ} \mathrm{C}$ and prior to cold shift at $20^{\circ} \mathrm{C}$. (A) Shared changes at $10^{\circ} \mathrm{C}$ between reil1-1 reil2-1 (DS2) and reil1-1 reil2-2 (DS1). $\log _{2}$-fold changes between mutants and Col-0 wild type in the 30S/40S fractions are calculated after normalization of protein LFQ-abundances by abundance sums of all detected 40 S RPs. Presence in mutants relative to absence in Col- 0 and $\log _{2}$-fold increases $>1$ are color-coded red, increases $<1$ are light red. Absence relative to presence in Col- 0 and $\log _{2}$-fold decreases $<-1$ are color-coded blue, decreases $>-1$ are light-blue. (B) Distribution of eIF3A1 LFQ-abundances across the sampled ribosome fractions and analyzed conditions (Experiment DS1, orange; experiment DS2 blue). (C) Distribution of eIF3C1 LFQ-abundances across the sampled ribosome fractions and analyzed conditions (Experiment DS1, orange; experiment DS2, blue). Note the abundance maxima in the 30/40S fractions that may contain $40 \mathrm{~S}$ subunits and $43 \mathrm{~S}$ pre-initiation complexes.

RPS24A (AT3G04920), RPS19A (AT3G02080), RPS25E (AT4G39200), and RPS15A (AT1G04270) increased consistently in the reil double mutants, RPS21B (AT3G53890) decreased (Fig. 9). Six additional 40S RPs changed inversely. Besides changes of structural 40S RPs, we found, at $10^{\circ} \mathrm{C}$, accumulation of eIF3A1 (AT4G11420), eIF3C1 (AT3G56150), and eIF3F1 (AT2G39990) in the non-translating 40S fraction of both double mutants (Fig. 11). Detection of eIF3C1 (AT3G56150) not fully specific and included a minority contribution by two of 16 peptides of eIF3C2 (At3g22860) in DS1 or one of 22 peptides in DS2 (Supplemental Table S6). These selected translation initiation factors are a subset of the ten detected components of the 13-subunit plant eukaryotic translation initiation factor 3 (eIF3) ${ }^{36}$. eIF3 is part of the $43 \mathrm{~S}$ and $48 \mathrm{~S}$ pre-initiation complexes and exits the mature complex upon joining of the $60 \mathrm{~S}$ subunit ${ }^{21}$.

Association of differential accumulation of RPs and RAPs in non-translating 405 and 605 fractions of reil double mutants with differential gene expression. With few exceptions, the differential accumulation of RPs in non-translating 40S and 60S fractions and the changes of ribosome biogenesis factors or eIF3 components in these fractions did not correlate with respective changes in transcript levels. We attempted to correlate differential accumulation of RPs in non-translating $40 \mathrm{~S}$ and $60 \mathrm{~S}$ fractions of reil double mutants from experiments DS1 and DS2 with differential accumulation of their transcripts at $20^{\circ} \mathrm{C}$ prior to cold shift and at 7 days after shift to $10^{\circ} \mathrm{C}$ (Supplemental Figure S9 A-B, E-F). In addition, we tested for time-shifted correlation of differential protein accumulation at 7 days after cold shift with preceding differential gene expression at 1 day after the shift (Supplemental Figure S9 C-D). With few exceptions, differential protein accumulation was not generally associated with changes of gene expression. However, the consistent decrease of $R P L 3 B$ (AT1G61580) gene expression (Fig. 7C) before and after cold shift, average $\log _{2}-\mathrm{FC}=-1.03\left(P=1.1510^{-7}\right.$ for the genotype effect according to 2-way ANOVA), matched to consistent reduction of RPL3B protein in the non-translating $60 \mathrm{~S}$ fraction of the two reil double mutants, experiments DS1 and DS2 (Fig. 9). In addition, an increase of RPL24C (AT2G44860) gene expression, average $\log _{2}-\mathrm{FC}=0.24\left(P=1.4310^{-2}\right.$ for the genotype effect according to 2-way ANOVA), matched to a consistent increase of RPL24C protein in the non-translating 60S fraction of the two reil double mutants. 


\section{Discussion}

The mixotrophic in vitro cultivation system: requirements and biases. We validated through this study the function of Arabidopsis REIL proteins as ribosome biogenesis factors of eukaryotic plant ribosomes. Our previous studies of REIL function in rosette leaves ${ }^{8}$ indicated that analyses of eukaryote ribosome complexes from leaf material were in part masked by almost equally abundant chloroplast ribosomes. Using root material, the organelle interference of UV-absorbance traces from density gradient separations of plant ribosome complexes was negligible (Fig. 1). Plastid and mitochondrial ribosomes co-purified from root material, but the prokaryote-type ribosome complexes were according to our proteome analyses on average more than 100 -fold less abundant than eukaryote ribosome complexes (Supplemental Figure S7, Supplemental Table S5). The choice of root material thus enabled relative and correlative quantification of eukaryote ribosome complexes (Figs. 2, 3). These analyses were not possible previously using leaf material ${ }^{8}$. Analyses of ribosomes complexes required amounts of $\sim 100 \mathrm{mg}(\mathrm{FW})$ tissue ${ }^{8}$. Our axenic in vitro cultivation system (Supplemental Figure S1) delivered required amounts of root tissue from plants that had developmental stage, $\sim 1.10^{16}$. Our current study confirmed reil gene expression in Col-0 wild type roots under our mixotrophic hydroponic cultivation conditions and strong reduction of expression of both genes in reil double mutants (Supplemental Figure S5). Recent promoter GUS studies confirmed constitutive reil2 expression throughout Arabidopsis seedlings under similar in vitro conditions and revealed relevance of REIL2 in the root organ by demonstrating high expression in the root tip?.

The previously reported delay of reil mutant development and growth in the cold attenuated using our cultivation system (Supplemental Figure S2-S3). We did not adjust temperature settings to achieve previous phenotypic strength. Instead, we used the phenotype-attenuating settings to minimize pleiotropic effects that may arise due to differential development of mutants and wild type (Supplemental Figure S2). We maintained comparability with our previous analyses of soil grown Arabidopsis rosettes ${ }^{6,8}$ by selecting stage $\sim 1.10$ plants to perform temperature shift experiments.

We were, however, confronted by a bias of mixotrophic cultivation. Mutant plants stayed dwarfed and flowered early even at optimized temperature without addition of sucrose to the liquid cultivation medium. As consequence, we cultivated in the presence of sucrose, but need to consider that the sucrose supply will mask or distort endogenous carbohydrate dependent signals. In addition, the illumination of the root system and the high humidity of hydroponic cultivation may affect transcriptional responses, for example, the observations linked to high light and reactive oxygen species that we report (Fig. 5A).

We discovered a further potential developmental bias of our study. Reil mutations affected root architecture (Supplemental Fig. S3). The branching pattern of hydroponic root systems in reil single and reil1 reil2 double mutants appeared changed. The functional link of REIL proteins to root development or root system architecture ${ }^{37}$ was supported further by functional enrichment analyses of differential gene expression. The reil1 reil2 double mutants had altered transcript levels of genes belonging to developmental growth ontologies, specifically root morphogenesis, epidermis and trichoblast differentiation (Supplemental Figure S6). These transcript changes between mutants and wild type became apparent already at optimized cultivation conditions and preceded cold induced responses. Because our current cultivation system was not suited for detailed root morphology or root patterning studies, we did not pursue this aspect further and considered this effect negligible for our current study.

We previously reported premature transcriptional cold acclimation responses at optimized temperature of reil1.1 reil2.1 rosette leaves from soil-cultivated plants ${ }^{8}$. An initial global differential gene expression analysis of shared significantly enriched GOs between the non-acclimated reil1 reil2 mutants and 7-days cold acclimated Col-0 appeared to confirm the triggering of premature transcriptional cold responses (Fig. 5B,C). The same comparison at single gene level, however, revealed an overlay of positively and negatively correlated transcriptional responses (Fig. 5D,E). Globally, premature responses to heat among the 138 genes of this ontology appeared to dominate over the cold responses of 263 genes, respectively, but neither cold acclimation nor heat acclimation responses were significantly enriched in the reil1 reil2 double mutants prior to cold shift (Fig. 5A). Selected marker genes of cold acclimation and cold response were either not prematurely changed, e.g. C-REPEAT/DRE BINDING FACTOR1/ DEHYDRATION-RESPONSIVE ELEMENT BINDING PROTEIN1B (CBF1/DREB1B), CBF2/DREB1C, CBF3/DREB1A, and VERNALIZATION INSENSITIVE 3 (VIN3), or had reduced expression prior to cold shift, e.g. COLD-INDUCED1 (KIN1) and KIN2 or COLD-REGULATED15A (COR15A) and COR15BA (Supplemental Figure S4). Cold acclimation of Arabidopsis has a well characterized carbohydrate component, where acclimating plants accumulate ${ }^{38,39}$ and de-acclimating plants rapidly reduce endogenous sugar levels $\mathrm{s}^{40}$. Adaptations in photosynthetic carbon metabolism provide signals that are integral part of Arabidopsis cold acclimation, including post transcriptional and transcriptional changes to sucrose metabolism ${ }^{41}$. In addition, photosynthesis-derived glucose is a regulator of TARGET OF RAPAMYCIN (TOR) signaling that is a component of Arabidopsis cold acclimation ${ }^{42,43}$. Considering the biases of our root cultivation system and especially the masking of carbohydrate signals, we cautiously conclude that the reil1 reil2 double mutants deregulate temperature responses in mixotrophic roots at optimized temperature.

REIL deficiency reveals requirement for de novo synthesis of eukaryote ribosomes after cold shift. Our current study confirmed and extended our previous observation that reil mutants delay accumulation of non-translating 60S LSUs after cold shift ${ }^{8}$. We demonstrated that the delay affects the non-translating 60 S LSUs and 40 S SSUs (Figs. 1,2). Shortage of non-translating free ribosome subunits was clearly transient and the balance of the abundances of non-translating large and small eukaryotic ribosome subunits was under tight control in plants (Fig. 3). We currently do not have evidence that control of this balance is dependent on REIL function in Arabidopsis. Our observation of concomitant decrease of 40 S SSUs in reil double mutants was 
unexpected, as the 40S SSU fraction increases in yeast $\Delta$ reil mutants ${ }^{2,4}$. Arabidopsis apparently has different control mechanisms to balance non-translating $40 \mathrm{~S}$ and $60 \mathrm{~S}$ subunits. However, our finding agrees with yeast data that show concomitant decreases of 40S SSUs and 60S LSUs in mutants that are defective in 60S structural RPs, whereas mutants defective in 40S structural RPs may accumulate high levels of $60 \mathrm{~S} \mathrm{LSUs}^{44}$.

We observed compensation responses of REIL deficiency that act both, before cold shift and after extended periods of cold (Figs. 2, 3, 4). All reil mutants over-accumulated baseline pools of non-translating 60S LSUs and $40 \mathrm{~S}$ SSUs, when not immediately responding to cold shift. This phenomenon may indicate either, over-accumulation of functional but yet non-translating subunits or may be reflect accumulation of non-functional ribosome subunits with assembly errors. These explanations are not distinguishable by our current experiments. Mutants containing a defective reil 2 gene depleted in addition the $80 \mathrm{~S}$ fraction in the cold. In Arabidopsis, the $80 \mathrm{~S}$ fraction similar to yeast ${ }^{17,18}$ appears to be composed of translating and non-translating $80 \mathrm{~S}$ ribosome monomers. Our $\mathrm{KCl}$-sensitivity tests of the Arabidopsis $80 \mathrm{~S}$ fraction and the poor correlation between $80 \mathrm{~S}$ and non-translating $60 \mathrm{~S}$ fractions indicated the heterogeneous nature of the $80 \mathrm{~S}$ fraction (Figs. 3, 4). Mainly for this reason, we did not analyze the ribo-proteome of the $80 \mathrm{~S}$ fraction in this study.

The function of the two REIL paralogs was clearly not fully redundant. REIL1 deficiency can be compensated successfully under both optimized and low temperature conditions, but a compensation response by over-accumulation of free subunits and slight deficiency after cold shift was observable (Fig. 2A-D). In contrast, REIL2 deficiency was successfully compensated only at optimized temperature, but compensation apparently failed in the cold. This may be indicated by persistent depletion of the $80 \mathrm{~S}$ fraction both in the reil 2 single mutants and in the reil1 reil2 double mutants (Fig. 2C).

Ribosome complex abundances recovered slowly in the cold shifted reil mutants. The time scale of 3-7 days after the shift is in agreement with previous reports of ribosome protein turnover in Arabidopsis ${ }^{45,46}$, where half-life of the ribosome population was approximately 3-4 days. Only RPP0D (MRT4-homolog, At1g25260) and the RACK1B and 1C paralogs were shorter lived with half-lives between 0.5 and 1.5 days ${ }^{46}$. Induction of $40 \mathrm{~S}$ and 60S RP transcripts after cold shift in the Col-0 wild type and compensatory enhanced and prolonged expression of these genes in reil1 reil2 double mutants indicate that Arabidopsis roots (Figs. 6, 7A,B) are prepared by transcript availability for ribosome de novo synthesis after cold shift. Rosette leaves provided similar observations at higher amplitude ${ }^{8}$.

Compensational depletion of non-translating ribosome complexes in the reil mutants cannot be explained alone by $60 \mathrm{~S}$ subunit shortage and deficient de novo ribosome biosynthesis. We propose that Arabidopsis maintains and controls pools of non-translating free 60S LSUs and 40S SSUs, likely under a tight and common control (Fig. 3). In addition a pool of assembled, but non-translating $80 \mathrm{~S}$ ribosomes appears to be present. These translationally inactive pools of ribosome complexes recycle ribosome complexes and may serve as buffers that respond to changing translation demands ${ }^{47-49}$. Such pools may save energy by harboring surplus extant ribosomes instead of initiating wasteful premature degradation. Such pools may accumulate, when translation demands are low and prepare plants for rapid responses to environmental cues without immediate need of comparatively slow de novo ribosome biosynthesis and assembly. Fluctuating temperature cues, such as investigated in this study, obviously alter translation demands.

Effects of REIL deficiency on translation initiation. In yeast cells, Reil deficiency, similar to other mutations that limit the amount of 60S LSU subunits, is associated to the hallmark observation of so-called halfmer polysomes ${ }^{2,5}$. Half-mers are polysome complexes with a stalled $40 \mathrm{~S}$ preinitiation complex and one or more fully assembled translating $80 \mathrm{~S}$ ribosomes bound to a single mRNA. Stalling of the preinitiation complex finds an obvious explanation through the lack of translationally competent 60S LSUs for translating 80S monomer assembly. Half-mer polysomes of yeast have been detected by sucrose density gradient centrifugation ${ }^{2,5}$. In our sucrose gradient analyses, we found indications of accumulating half-mer polysomes in the reill reil2 double mutants at 1-3 days after cold shift (Fig. 1), but this phenomenon did not persist after prolonged cold exposure (Figs. 1, 4). Proteomic analysis demonstrated the presence of components of the eIF3 multi-protein complex ${ }^{20}$ in the non-translating $40 \mathrm{~S}$ fraction and in a subsequent fraction also enriched for organelle 50S RPs. These observations likely indicate the presence of co-purified plant $43 \mathrm{~S}$ and $48 \mathrm{~S}$ preinitiation complexes, respectively (Fig. 11B,C). Accumulation of eIF3 components in both cold acclimating reil1 reil2 double mutants indicated accumulation mostly of stalled $43 \mathrm{~S}$ preinitiation complexes after cold shift (Fig. 11A-C). The existence of halfmer polysome complexes in cold exposed reil double mutants was supported by the presence of eIF3A1 in the mutant low-oligomer polysome fraction in one of our analyses (Fig. 11B). We conclude that REIL protein deficiency can cause accumulation of Arabidopsis $43 \mathrm{~S}$ preinitiation complexes and that half-mer polysomes can accumulate. These observations support REIL function as a ribosome biogenesis factor by indicating lack of translationally competent $60 \mathrm{~S}$ subunits in the cold exposed mutants.

At transcriptome level, we found evidence that reil deficiency feeds back constitutively onto gene expression of the eIF3C2 paralog. REIL deficiency specifically released the suppression of eIF3C2 expression (Fig. 8) and largely did not affect expression of other constituents of the eIF3 complex. In our current study, the eIF3C2 protein was not selectively detectable with one of 22 or two of 16 detected peptides shared between the eIF3C1 and eIF3C2 paralogs (Supplemental Table S6). The 805 amino acid eIF3C2 protein has an internally shortened $\mathrm{N}$-terminal domain and lacks a large part of the C-terminus compared to the 900 amino acid eIF3C1 paralog ${ }^{20}$. It may be allowed to speculate that the eIF3C2 paralog competes with eIF3C1 in plant eIF3 complexes and/ or may have an inhibitory regulatory role that adjusts eIF3 abundance to availability of translationally competent $60 S$ LSUs. Such a function may cause a sub-fraction of eIF3 not to bind to ribosomes, but a mechanistic explanation must currently remain elusive. 
Effects of REIL deficiency on ribosome biogenesis. The reil1 reil2 mutants accumulate Arabidopsis homologs of yeast $60 \mathrm{~S}$ and $66 \mathrm{~S}$ ribosome biogenesis factors in the non-translating $60 \mathrm{~S}$ fraction. Homologs of cytosolic biogenesis factors, NMD3, TIF6, RLP24 that are assembled within the nucleus and released in the cytosol before the 60S LSU becomes translationally competent accumulate before and after temperature shift (Fig. 10A-E). Nuclear ribosome biogenesis factors associate predominantly with the $66 \mathrm{~S}$ pre-ribosome. These factors include proteins that block positions in the $66 \mathrm{~S}$ complex which are later occupied by the cytosolic biogenesis factors in the $60 \mathrm{~S}$ pre-ribosome. Nuclear ribosome biogenesis factors accumulated in the cold in both reil double mutants and only in one double mutant, reil1.1 reil2.2 before cold shift (Fig. 10A). These observations indicate a likely heterogeneity between the reil2 alleles that are currently available.

We conclude that REIL deficiency inhibits the release of Arabidopsis NMD3-, TIF6-, and RLP24-homologs and thereby slows down biogenesis of translationally competent 60S LSUs. We interpret accumulation of nuclear ribosome biogenesis factors as pile-up of precursor complexes caused by blocked cytosolic $60 \mathrm{~S}$ maturation. We observe that the block of $60 \mathrm{~S}$ maturation intensifies at low temperature (Fig. 10). Taken together the observations prove conserved REIL function as a cytosolic 60S biogenesis factors. In addition, we imply previously non-described plant proteins, namely a $\mathrm{R} 3 \mathrm{H}$-domain protein and a LEA-like protein that do not have apparent homology in yeast or humans beyond the conserved $\mathrm{R} 3 \mathrm{H}$-domain, to likely contribute to plant specific aspects of $60 \mathrm{~S}$ biogenesis.

At transcriptome level, we find NUC2, but not NUC1 gene expression constitutively activated. NUC2 codes for one of the two antagonistic plant nucleolins and thereby provides a functional link to eukaryote ribosome biogenesis. Both, NUC2 and NUC1 expression are required for plant growth. NUC2 and NUC1 act as histone chaperons early in eukaryotic ribosome biogenesis. The nucleolins control rDNA variant expression by modulation of $45 \mathrm{~S}$ rRNA transcription and/ or processing ${ }^{24,25}$. NUC2 expression is high in the root apical meristem, similar to our still enigmatic finding of reil dependent PISTILLATA deregulation in roots. Both nucleolins are expressed in root and leaf tissues, but NUC2 transcripts accumulate more in root than in leaf and shoot tissue ${ }^{24}$. Durut and co-authors conclude that NUC2 might bind nucleosomes to induce and/or maintain a repressive rDNA chromatin state ${ }^{24}$. Building on the hypothesis that NUC2 may act as a repressor ${ }^{25}$, we may naively interpret our observation of deregulated NUC2 expression as indication of a feedback repression of the primary step of rRNA transcription in response to blocked 60S maturation. However, studies on reil2 single paralog mutants suggest that the control of rRNA transcription may be negligible compared to feedback control of rRNA processing exerted downstream of the $35 \mathrm{~S}$ rRNA precursor ${ }^{9}$. Obviously, this control affected both of the two parallel terminal plant pre-rRNA processing pathways ${ }^{50}$. Accumulation of pre-60S maturation factors confirmed stalling of nuclear $66 \mathrm{~S}$ maturation complexes that contain partially processed rRNA.

REIL deficiency affects the RP paralog composition of non-translating 40S SSU and 60S LSU fractions. We discovered that REIL deficiency affects the paralog composition of non-translating ribosome subunits. Most of our observations are less than twofold changes (Fig. 9). Considering the composite nature of the 40S SSU and 60S LSU fractions that may contain both, recycled ribosome populations that were synthesized prior to cold shift and ribosome populations that are de novo synthesized in the cold, we were surprised by these observations that indicate ribosome heterogeneity ${ }^{14}$ and were consistent between the two reil double mutants.

One of the more obvious observations was the consistent decrease of RPL24B of the eL24 RP family in the non-translating $60 \mathrm{~S}$ fractions of the reil1 reil2 double mutants (Fig. 9). This phenomenon can be linked directly to the concomitant accumulation of the RPL24C (Fig. 10), the Arabidopsis homolog of the yeast RLP24 maturation factor that is a placeholder during pre-60S complex assembly and is replaced in the cytosol to form translationally competent 60S LSUs ${ }^{2}$. Competent 60S LSUs of yeast contain either yeast RPL24A or RPL24B. Similar to yeast, Arabidopsis REIL proteins appear to be required to facilitate or accelerate this exchange.

Two other 60S RP families are assembled onto the 60S subunit in the cytosol, uL10 and uL16 ${ }^{2}$. Of these families, we find RPP0B, uL10 family, to be consistently decreased at $10{ }^{\circ} \mathrm{C}$ and inversely increased at $20^{\circ} \mathrm{C}$ in both reil double mutants (Fig. 9). RPP0B is one of the Arabidopsis paralogs that code for the P-stalk protein $\mathrm{P} 0$ that anchors the P-stalk to the ribosome ${ }^{51-54}$. P0 interacts as a scaffold of other P-stalk proteins of the P1/P2 family, here represented by consistent abundance changes of Arabidopsis RPP1A, RPP1B and RPP1C (Fig. 9) and overall reduced abundance of RPP2D in the reil double mutants (Supplemental Figure S8A). Only the fully assembled and active P-stalk substructure interacts with translation elongation factors and is required for translation.

Besides these changes of ribosome subunit composition, we found temperature dependent and independent effects on the composition of structural ribosome proteins within the non-translating 40S SSU and 60S LSU (Fig. 9). Such compositional changes must involve either an indirect REIL function that affects de novo subunit assembly in the nucleus or may involve RP paralog exchange of assembled ribosome that pre-exist in the cytosol prior to cold shift. Such a mechanism was previously proposed for in situ ribosome repair ${ }^{55}$.

Among the many observations of compositional changes, we would like to highlight the effect on the uL3 family that is part of 60 S LSUs ${ }^{56}$. REIL proteins apparently are required to accumulate RPL3B paralogs in nontranslating 60S fractions (Fig. 9). The slight accumulation of abundant RPL3A, an essential gene for Arabidopsis ${ }^{57}$, appears to compensate reduction of RPL3B in our study. In addition, RPL3B decrease in the absence of REIL proteins has a transcriptional component, as the reduction of $R P L 3 B$ transcript is one of the rare observations of otherwise absent associations between transcriptome and proteome changes of non-translating fractions (Supplemental Figure S9). 


\section{Conclusion}

Our study proves that Arabidopsis REIL proteins function as cytosolic ribosome biogenesis factors and appears to further support their role of accelerating ribosome de novo synthesis in the cold. We suggest that Arabidopsis compensates for the lack of REIL function at optimized temperature, but cannot fully compensate in the cold. The compensation mechanism may involve accumulation of non-translating pools of ribosome complexes and appears to activate pre-existing pools upon cold-shift. We hypothesize, that Arabidopsis controls non-translating ribosome complexes to buffer fluctuating demands on translation. Thereby Arabidopsis may limit the demand for de novo ribosome synthesis under rapidly changing environmental conditions. REIL function is mostly post-transcriptional, however, with a few apparent transcriptional components. REIL function appears to feed forward onto formation of $43 \mathrm{~S}$ initiation complexes likely in a passive mode by modulating 60S LSU availability or more actively via eIF3C2, NUC2 or RP gene expression and feedback onto nuclear pre-60S subunit assembly. Through potential disturbance of de novo ribosome biosynthesis and translation initiation, reil mutants allow the study of these processes and their control in plants. Alternatively, our results may indicate accumulation of defective or misassembled ribosomes resulting from lack of a proposed REIL proofreading function ${ }^{1,2}$. This interpretation still presumes de novo synthesis of ribosomes after cold shift and assumes a specific requirement for REIL proof reading in the cold.

Unexpectedly, REIL function in Arabidopsis appears to control the paralog composition of non-translating $40 \mathrm{~S}$ and $60 \mathrm{~S}$ subunits. Considering paralog composition as one mechanism that may create heterogeneous functional ribosome populations ${ }^{58-61}$, we propose that reil mutants and temperature acclimation cues may provide feasible and highly relevant experimental systems to study ribosome heterogeneity in plants ${ }^{14}$. Clearly, further studies are required with a focus on functional ribosomes to extend and substantiate our current observations of non-translating ribosome heterogeneity and to solve the question whether ribosome accumulation in reil mutants compensates or reflects accumulation of misassembled ribosomes.

\section{Materials and methods}

Plant material. The single-paralog mutants, reil1-1, reil2-1, and reil2-2 lines, with T-DNA insertions in exon 2 at base pairs 475,733, and 731, respectively, were selected to investigate the role of REIL proteins for the plant growth in suboptimal temperature condition. All T-DNA insertions were characterized previously by sequencing of the left border of insertion sites ${ }^{6}$. The double homozygous reill-1 reil2-1 (DKO1) mutant ${ }^{6}$ was created by crossing the T-DNA insertion mutant SALK_090487 (reil1-1) that was obtained through the Nottingham Arabidopsis Stock Centre ${ }^{62}$ and GK_166C10 (reil2-1) of the GABI-Kat program ${ }^{63}$. The second allelic double homozygous reil1-1 reil2-2 (DKO2) mutant was created in this study by crossing the T-DNA insertion mutant SALK_090487 (reil1-1) and reil2-2 (SALK_040068). Homozygosity of the insertion sites was verified by PCR amplification of genomic DNA with the previously described T-DNA- and reil1- and reil2-specific oligonucleotides $^{6}$. All system-profiling analyses were performed with the two double mutants and controls of the ecotype Arabidopsis thaliana Col-0 (wild type), i.e. the common genetic background of all mutants in this study. The three single-paralog reil mutants, reil1-1, reil2-1 and reil2-2, were subjected to comparative analysis of ribosome complexes.

Photographic documentation. The Arabidopsis shoot and root systems were digitally photographed at 300 dpi horizontal and vertical resolution using a Nikon D5100 camera with up to $4,928 \times 3,264$ pixels or a NIKON D850 camera with up to $8,256 \times 5,504$ pixels. Photographs were cropped to size and resolution reduced to meet size limitations of composite figures using Adobe Photoshop CS5 Extended (Version 12.0.4 $\times 64$ ). The scale bars were manually added using external documentation of scale and magnification.

Hydroponic system for growth, controlled temperature shift and sampling of Arabidopsis root materials. Generation of seed batches, storage, sterilization, and in vitro pre-cultivation were as described previously ${ }^{6}$. The hydroponic growth system for Arabidopsis plants used circular white glass containers with glass lids ${ }^{10}$. The containers had a slightly tapered bottom with $10.0 \mathrm{~cm}$ top diameter and $7.5 \mathrm{~cm}$ bottom diameter (Supplemental Fig. S1). The containers had a total volume of $\sim 580 \mathrm{~mL}$. Each glass contained $250 \mathrm{~mL}$ of liquid MS media with $2 \%$ sucrose (w/v) adjusted to $\mathrm{pH} 5.7^{15}$. Sucrose addition reduced developmental and growthdifferences between mutants and Col-0 wild type. The liquid was first poured into the jar, then a sterilized stainless steel wire mesh insert of $9 \mathrm{~cm}$ diameter, with mesh size $1.40 \mathrm{~mm}$ and wire thickness $0.25 \mathrm{~mm}$ was adjusted to the top of the liquid (Supplementary Fig. S1). Four small $\sim \mathrm{cm}^{3}$-blocks of solid MS media that contained $0.8 \%(\mathrm{w} / \mathrm{v})$ agar were placed on top of the mesh before applying single sterilized Arabidopsis seeds to each block. Four plants were routinely cultivated per container. The containers were closed tightly, but not airtight. Glass lids were fixed to the container by stainless steel clamps before transfer to a controlled-environment chamber for growth. The seeds were allowed to germinate and grow under sterile conditions in a long day photoperiod with a $16 \mathrm{~h} / 8 \mathrm{~h}$-day/night cycle. External temperature settings of the chamber were controlled. External light intensity was $100-150 \mu \mathrm{mol}$ photons $\mathrm{m}^{-2} \mathrm{~s}^{-1}$. External relative humidity was $60-70 \%$. The standard temperature regime was $20^{\circ} \mathrm{C}$ during the day and $18^{\circ} \mathrm{C}$ during night (Supplementary Fig. S1).

Temperature shift experiments and root harvests. Plants were pre-cultivated at $20^{\circ} \mathrm{C}$ standard conditions to developmental stage $\sim 1.10^{16}$. At this stage, the plants were transferred carefully within their hydroponic containers from standard conditions to a second controlled-environment chamber with $10{ }^{\circ} \mathrm{C}$ temperature during the day and $8{ }^{\circ} \mathrm{C}$ during the night, but otherwise equal external light cycle, light intensity, and relative humidity settings (Supplemental Fig. S2). Non-cold shifted roots were harvested immediately before temperature shift and are in the following denoted non-acclimated or 0 day samples. Subsequently, samples 
were harvested after one day, three days, one week, and three weeks after cold shift (Supplemental Fig. S3). Each harvested biological replicate was the pool of total root material from four plants of a single container. Three biological replicates, $n=3$, were harvested for microarray based transcriptome analyses. Five replicates per experimental condition, $\sim 20$ individual root systems, were pooled into single samples for the analysis of ribosome complexes using sucrose density gradients.

Sucrose density gradient analysis of ribosome preparations from Arabidopsis root tissue. Ribosome fractions from Arabidopsis Col-0 wild type and reil mutants were prepared from pools of hydroponically grown root systems as described previously ${ }^{8,10}$. Due to the complex procedure, single pools of non-acclimated roots and roots at 1 day, 3 days, 7 days, and 21 days after cold-shift were analyzed per genotype for qualitative analyses. Briefly, per sample 100-102 mg fresh weight (FW) of frozen root tissue was homogenized $30 \mathrm{~min}$ on ice with $0.5 \mathrm{~mL}$ polysome extraction buffer (PEB) in horizontal tubes on an orbital shaker set to 400 rotations per minute. The final PEB buffer composition was $200 \mathrm{mM}$ Tris- $\mathrm{HCl}$ adjusted to $\mathrm{pH} 9,200 \mathrm{mM}$ $\mathrm{KCl}, 25 \mathrm{mM}$ EGTA, $36 \mathrm{mM} \mathrm{MgCl}_{2}, 5 \mathrm{mM}$ DTT, $50 \mu \mathrm{g} \mathrm{mL}^{-1}$ cycloheximide (CHX), $50 \mu \mathrm{g} \mathrm{mL}^{-1}$ chloramphenicol (CPL), $1.0 \mathrm{mg} \mathrm{mL}^{-1}$ heparin, $1 \%$ (v/v) Triton X-100, 1\% (v/v) Tween 20, 1\% (w/v) Brij-35, 1\% (v/v) IGEPAL CA-630, $1 \%(\mathrm{v} / \mathrm{v})$ polyoxyethylene, $1 \%(\mathrm{w} / \mathrm{v})$ deoxycholic acid, $1 \mathrm{mM}$ phenylmethylsulfonylfluoride (PMSF), and the equivalent of 0.002 tablets $\mathrm{mL}^{-1} \mathrm{cOmplete}$ protease inhibitor mixture. All chemicals were obtained from Sigma-Aldrich (Taufkirchen, Germany). The following procedures were carried out at $4{ }^{\circ} \mathrm{C}$. After initial centrifugation at 14,000 $\mathrm{g}$ for $10 \mathrm{~min}$, the extract with remaining debris was placed on top a QIAshredder spin column of the RNeasy Plant Mini Kit (Qiagen, Hilden, Germany) and centrifuged again at 14,000 g for 1 min to remove all cell debris. Volumes of $0.5 \mathrm{~mL}$ of extract were loaded onto $9 \mathrm{~mL}$ of a $15 \%(\mathrm{w} / \mathrm{v})$ to $60 \%$ (w/v) sucrose gradient that was prepared in PEB without detergents, heparin and protease inhibitors. The loaded density gradients were centrifuged at $33,000 \mathrm{~g}$ for $14.5 \mathrm{~h}$ at $4{ }^{\circ} \mathrm{C}$ using a six-position SW41 Ti rotor (Beckman Coulter, Krefeld, Germany). The positions of ribosome complexes varied slightly with each centrifugation run that comprised a set of six samples. In each centrifugation run, a non-sample control gradient was loaded with $0.5 \mathrm{~mL}$ PEB and used for photometric base line subtraction. The five remaining positions were used for root samples and included an Arabidopsis Col-0 sample for the estimation of relative abundances of ribosome complexes. Five centrifugation runs contained samples that were harvested at the same experimental time point. In the sixth centrifugation run, a randomly selected mutant of each time point was analyzed. The sucrose density gradients were separated into $\sim 40$ fractions of approximately $250 \mu \mathrm{L}$ per fraction using a programmable density gradient fractionation system (Teledyne Isco Inc., NE, USA). An absorbance profile was recorded continuously at $254 \mathrm{~nm}$ wavelength using a 15\% (w/v) sucrose solution in PEB without detergents, heparin and protease inhibitors, prior to each gradient recording to calibrate the absorbance minimum ( $0 \%$ absorbance $\left.{ }_{254 \mathrm{~nm}}\right)$. The absorbance profiles were exported from the acquisition system and background subtracted by the profile of blank sample of each centrifugation run and identically scaled. Abundances of ribosome fractions were measured as background subtracted peak areas (Supplemental Table S1). Peak areas and the total sum of all peak areas including the 40S, $60 \mathrm{~S}, 80 \mathrm{~S}$ and polysome fractions, were determined by Chromas Lite 2.1 software (http://chromas-lite.software. informer.com/2.1/).

$\mathrm{KCl}$-sensitivity test of ribosome complexes. Exemplary in vitro KCl-sensitivity of translating ribosome complexes was performed with wild type root tissue compared the reil1-1 reil2-1 double mutant at 21 days after cold shift as a compromise between immediate and prolonged cold exposure. A pooled root sample of each simple type was split into two technical replicates of $100 \mathrm{mg} \mathrm{FW}$ each. Each of the replicates was extracted with $0.5 \mathrm{~mL}$ PEB that either contained regular $200 \mathrm{mM} \mathrm{KCl}$ or elevated $400 \mathrm{mM} \mathrm{KCl}$.

After $30 \mathrm{~min}$ incubation on ice all samples were fractionated by $15-60 \%$ sucrose density gradients. All samples were centrifuged in a single $2 \mathrm{~h}$ run with sedimentation at $50,000 \mathrm{~g}$ and $4{ }^{\circ} \mathrm{C}$ using small $13 \times 51 \mathrm{~mm}$ ultracentrifugation tubes in SW55 Ti rotor (Beckman Coulter, Krefeld, Germany). These centrifugation settings were optimized for polysome and monosome separations. Abundance analysis of the separated ribosome complexes was as described above omitting fraction collection.

Transcriptome analysis. Twenty seven root samples, 3 experimental conditions $\times 3$ genotypes $\times 3$ biological replicates, of non-acclimated and 1 day or 7 days cold acclimating Col-0, reil1-1 reil2-1, reil1-1 reil2-2 were analyzed. Liquid nitrogen frozen samples of 25.1-26.8 mg fresh weight were ground to fine powder. Total RNA was extracted from frozen powder using the RNeasy Plant Mini Kit and RNase-free DNase (Qiagen, Limburg, Netherlands) according to the manufacturer's instructions as was described previously ${ }^{8}$ with down-scaling modifications. Briefly, $110 \mu \mathrm{L}$ RNA extraction buffer was used and $30 \mu \mathrm{L}$ of RNase-free water for RNA elution. Total RNA was quantified by NanoDrop ${ }^{\text {Tw }}$ spectrophotometer (ThermoFisher Scientific, Germany) using absorbance wavelength $260 \mathrm{~nm}$. RNA quality was evaluated by formaldehyde agarose gel electrophoresis and $\mathrm{OD}_{260 / 280}$ and $\mathrm{OD}_{260 / 230}$ ratios. The RNA integrity number (RIN) of RNA samples was assessed using an Agilent 2100 Bioanalyzer (Agilent Technologies, Germany). RNA samples of $10-15 \mu \mathrm{L}$ per sample with RIN $>8$ were processed by ATLAS Biolabs GmbH (Berlin, Germany) using Agilent Feature Extraction software (v10.7). The obtained gene expression data sets were quantile normalized using the normalize.quantile routine of the preprocessCore $\mathrm{R}$ statistical computing and graphics package (https://www.r-project.org/). The obtained 4X44K Agilent microarray data comprise variable numbers of redundant gene probes. The expression data sets are available from the Gene Expression Omnibus (https://www.ncbi.nlm.nih.gov/geo/) through accession GSE144916. Gene expression information of redundant probes was averaged to reduce bias for functional enrichment analyses. Gene expression information was normalized per gene model to the average expression value from non-acclimated Col-0. Alternatively, the differential gene expression of mutants over Col-0 was calculated at each time point. 
Averaged $\log _{2}$-transformed ratios of differential gene expression, standard errors and statistical test results are reported in the supplement (Supplemental Table S2).

Functional enrichment analyses of gene expression data. Functional enrichment analysis of differential gene expression was carried out by parametric analysis of gene set enrichment ${ }^{19,64}$ using the agriGO v2.0 web-service at http://systemsbiology.cau.edu.cn/agriGOv2/. The parametric analysis of gene set enrichment processes lists of genes with $\log _{2}$-transformed numerical differential gene expression values from one or more experimental conditions and provides information on the enrichment of expression increases or decreases across gene sets of at least 10 gene entries that are defined by 2,145 GO gene ontology (GO) terms. Enrichment of mean $\log _{2}$-fold changes $\left(\log _{2}\right.$-FC) were evaluated by z-scores, and by false discovery rate (FDR)-adjusted $P$ values $^{65}$ applying a $P<0.05$ threshold (Supplemental Table S3).

Proteome analysis of ribosome preparations from Arabidopsis root tissue. We performed two complex experiments of ribosome fraction preparation and shotgun ribo-proteome analysis that resulted in data sets DS1 and DS2, respectively. Each data set contained a pooled Arabidopsis Col-0 root sample and a pooled double mutant root sample either of reil1-1 reil2-2 (DS1) or of reil1-1 reil2-1 (DS2). Samples prior to temperature shift and samples prepared at 7 days after $10^{\circ} \mathrm{C}$ cold shift were analyzed.

Sucrose density fractionation and subsequent proteomic analysis were performed with approximately equal amounts of $100 \mathrm{mg}$ FW. Tryptic peptides were prepared from up to five non-translating ribosome fractions and a low-oligomer polysome fraction by filter aided sample preparation ${ }^{66,67}$. Sucrose density gradient fractions were washed repeatedly with $500 \mu \mathrm{L}$ of $0.04 \mathrm{M}$ Tris-HCL buffer ( $\mathrm{pH} 8.4$ ) with $0.2 \mathrm{M} \mathrm{KCl}$ and $0.1 \mathrm{M} \mathrm{MgCl}_{2}$ using regenerated cellulose membranes, Amicon Ultra- 0.5 centrifugal filter units, with a $3 \mathrm{kDa}$ molecular size cutoff (Merck, Kenilworth, New Jersey, USA). Removal of sucrose was considered complete, when the $500 \mu \mathrm{L}$ volume decreased to below $100 \mu$ within $10 \mathrm{~min}$ of centrifugation at $5000-7000 \mathrm{rpm}$ and $4{ }^{\circ} \mathrm{C}$.

Shotgun proteome analysis of recovered peptides was performed by liquid chromatography-tandem mass spectrometry (LC-MS/MS) with an ACQUITY UPLC M-Class system (Waters Corporation, Milford, MA, USA) hyphenated to a Q-Exactive HF high-resolution mass spectrometer (Thermo Fisher Scientific, Waltham, MA, USA). Samples were separated by reverse-phase nano-liquid chromatography using a 125/ $132 \mathrm{~min}$ (DS1/ DS2) $3 \%$ to $85 \%(\mathrm{v}: \mathrm{v})$ acetonitrile $(\mathrm{ACN})$ gradient. Mass spectrometric data acquisition was performed by data dependent top- $\mathrm{N}$ tandem mass spectrometry (dd-MS2). This acquisition mode fragmented the top 10/ 15 (DS1/ DS2) most intense ions per full scan. Full scans were acquired at a resolution of 120,000/ 60,000 (DS1/ DS2) with automatic gain control (AGC) target set to 3e6/ 1e6 (DS1/ DS2), maximum injection time 100/ $75 \mathrm{~ms}$ (DS1/ DS2), and scan range 300 to $1600 \mathrm{~m} / \mathrm{z}$ in profile mode. Each dd-MS2 scan was recorded in profile mode at a resolution of 15,000/30,000 (DS1/ DS2) with AGC target set to 1e5, maximum injection time $150 \mathrm{~ms}$, isolation window of $1.2 / 1.4 \mathrm{~m} / \mathrm{z}$ (DS1/ DS2), normalized collision energy $27 \mathrm{eV}$ and dynamic exclusion of $30 \mathrm{~s}$. The LC-MS/MS files were analysed by MaxQuant software (Version 1.6.0.16), http://www.coxdocs.org/doku. php?id=maxquant:common:download_and_installation. Arabidopsis thaliana FASTA files of the reviewed SwissProt compendium in the UniProt database ${ }^{68}$ served peptide annotation. All peptides, including unique peptides and non-specific peptides of highly similar or identical paralogs of structural cytosolic ribosome proteins were analyzed by label-free quantification (LFQ) ${ }^{69}$.

The proteomics data set, identifier PXD016292, and detailed experimental settings are available via the ProteomeXchange Consortium at the PRIDE partner repository, https://www.ebi.ac.uk/pride/archive/login ${ }^{70}$. Proteins were annotated according to majority peptide identification. Specificity of detected peptides for cytosolic RP paralogs and ribosome-associated proteins (RAPs) was analyzed including potential non-specificity by minority peptide calls. Supplemental Table S4 reports proteomic fraction characterization and the subset of samples from Pride data set PXD016292 used in this study. We identified the 40S and 60S RPs, and the organelle $30 \mathrm{~S}$ and $50 \mathrm{~S}$ RPs according to previous publications ${ }^{71-75}$. Due to the differences of cytosolic ribosome subunits in each fraction, and to account for the variation between sample types, we normalized protein LFQ-abundances in fractions that contained 60S RPs to the sum of LFQ-abundances of all detected 60S proteins in each of the respective fractions. This procedure allowed analysis of compositional changes of $60 \mathrm{~S}$ ribosome complexes, but may create false positives of components due to co-purified organelle RPs or other proteins. For this reason, we only considered changes relevant, if the non-normalized LFQ abundance had a maximum in the 60S or 60S/80S fractions and not in the 30S/40S or 50S fraction. We excluded all 40S RPs from the compositional analysis of the non-translating $60 \mathrm{~S}$ fractions. To assess compositional changes of non-translating $40 \mathrm{~S}$ complexes we normalized to the sum of all 40S RPs in each fraction. We excluded all 60S RPs and only considered proteins that were more abundant in the $30 \mathrm{~S} / 40 \mathrm{~S}$ fraction relative to the $60 \mathrm{~S}$ fraction. Due to the overall experimental design, we did not apply statistical analyses for feature selection. Instead, we selected for changes that were shared between the reil1 reil2 double mutants at $10^{\circ} \mathrm{C}$. In a more stringent analysis, we selected the respectively shared changes at both temperatures $10^{\circ} \mathrm{C}$ and $20^{\circ} \mathrm{C}$.

Association analysis of changes in transcript and relative protein abundance. The differential transcript levels of the reil1 reil2 mutants relative to Arabidopsis thaliana Col-0 wild type were compared to changes of relative protein abundance in the non-translating $40 \mathrm{~S}$ and $60 \mathrm{~S}$ plus $60 \mathrm{~S} / 80 \mathrm{~S}$ fractions of the proteome analysis (Supplemental Figure S9). For this purpose, we correlated the differential accumulation of cytosolic RPs and RAPs in non-translating ribosome fractions reported in Supplemental Table S6 to differential gene expression (Supplemental Table S2, columns X to AC). We merged transcript and protein information according to the reported gene model information of the transcriptome and proteome analyses (Pride data set PXD016292). Note that the transcript information was in part splice variant specific. The proteome analyses in many cases did not 
distinguish splice variants and contained non-specific information due to peptides of proteins from the highly similar or in part identical ribosome protein families (Supplemental Table S6).

Statistical analyses and data visualizations. Quantile-normalized $\log _{2}$-transformed ratios of DEGs (differentially expressed genes) or $\log _{2}$-transformed of differential protein abundances were analyzed, correlated and visualized by Microsoft Excel software of the Office Professional Plus 2010 package (Microsoft) and the Multiple Experiment Viewer version 4.9.0 (http://www.mybiosoftware.com/mev-4-62-multiple-experiment-viewe r.html). Significance thresholds $(P)$ of statistical analyses, e.g. one- or two-way analyses of variance (ANOVA), and two-group comparisons by heteroscedastic Student's $t$ test, were routinely $P<0.05$ if not mentioned otherwise. Details of the data analysis and visualization methods are reported in the legends of figures and tables.

Received: 6 November 2020; Accepted: 6 January 2021

Published online: 28 January 2021

\section{References}

1. Greber, B. J., Boehringer, D., Montellese, C. \& Ban, N. Cryo-EM structures of Arx1 and maturation factors Reil and Jjj1 bound to the 60 S ribosomal subunit. Nat. Struct. Mol. Biol. 19, 1228-1234 (2012).

2. Greber, B. J. et al. Insertion of the biogenesis factor Reil probes the ribosomal tunnel during 60S maturation. Cell 164, 91-102 (2016).

3. Iwase, M. \& Toh-e, A. Ybr267w is a new cytoplasmic protein belonging to the mitotic signaling network of Saccharomyces cerevisiae. Cell Struct. Funct. 29, 1-15 (2004)

4. Lebreton, A. et al. A functional network involved in the recycling of nucleocytoplasmic pre-60S factors. J. Cell Biol. 173, 349-360 (2006).

5. Parnell, K. M. \& Bass, B. L. Functional redundancy of yeast proteins Reh1 and Rei1 in cytoplasmic 60S subunit maturation. Mol. Cell Biol. 29, 4014-4023 (2009).

6. Schmidt, S., Dethloff, F., Beine-Golovchuk, O. \& Kopka, J. The REIL1 and REIL2 proteins of Arabidopsis thaliana are required for leaf growth in the cold. Plant Physiol. 163, 1623-1639 (2013).

7. Schmidt, S., Dethloff, F., Beine-Golovchuk, O. \& Kopka, J. REIL proteins of Arabidopsis thaliana interact in yeast-2-hybrid assays with homologs of the yeast Rlp24, Rpl24A, Rlp24B, Arx1, and Jjj1 proteins. Plant Signal Behav. 9, e28224 (2014).

8. Beine-Golovchuk, O. et al. Plant temperature acclimation and growth rely on cytosolic ribosome factor homologs. Plant Physiol. 176, 2251-2276 (2018).

9. Yu, H. et al. STCH4/REIL2 confers cold stress tolerance in Arabidopsis by promoting rRNA processing and CBF protein translation. Cell Reports 30, 229-242 (2020).

10. Firmino, A. A. P. et al. Separation and paired proteome profiling of plant chloroplast and cytoplasmic ribosomes. Plants 9,892 (2020).

11. Cook, D., Fowler, S., Fiehn, O. \& Thomashow, M. F. A prominent role for the CBF cold response pathway in configuring the lowtemperature metabolome of Arabidopsis. PNAS 101, 15243-15248 (2004).

12. Maruyama, K. et al. Identification of cold-inducible downstream genes of the Arabidopsis DREB1A/CBF3 transcriptional factor using two microarray systems. Plant J. 38, 982-993 (2004).

13. Van Buskirk, H. A. \& Thomashow, M. F. Arabidopsis transcription factors regulating cold acclimation. Physiol. Plant. 126, 72-80 (2006).

14. Martinez-Seidel, F., Beine-Golovchuk, O., Hsieh, Y.-C. \& Kopka, J. Systematic review of plant ribosome heterogeneity and specialization. Front. Plant Sci. 11, 948 (2020).

15. Murashige, T. \& Skoog, F. A. Revised medium for rapid growth and bio assays with tobacco tissue cultures. Physiol. Plant. 15, 474-497 (1962).

16. Boyes, D. C. et al. Growth stage-based phenotypic analysis of Arabidopsis: a model for high throughput functional genomics in plants. Plant Cell 13, 1499-1510 (2001).

17. Martin, T. E. \& Hartwell, L. H. Resistance of active yeast ribosomes to dissociation by KCl. J. Biol. Chem. 245, 1504-1506 (1970).

18. Zylber, E. A. \& Penman, S. The effect of high ionic strength on monomers, polyribosomes, and puromycin-treated polyribosomes. Biochim. Biophys. Acta 204, 221-229 (1970).

19. Tian, T. et al. AgriGO v2.0: a GO analysis toolkit for the agricultural community, 2017 update. Nucleic Acids Res. 45, W122-W129 (2017).

20. Burks, E. A., Bezerra, P. P., Le, H., Gallie, D. R. \& Browning, K. S. Plant initiation factor 3 subunit composition resembles mammalian initiation factor 3 and has a novel subunit. J. Biol. Chem. 276, 2122-2131 (2001).

21. Browning, K. S. \& Bailey-Serres, J. Mechanism of cytoplasmic mRNA translation. Arabidopsis Book 13, e0176 (2015).

22. Brady, S. M. et al. A high-resolution root spatiotemporal map reveals dominant expression patterns. Science 318, 801-806 (2007).

23. Lee, J.-Y. et al. Transcriptional and posttranscriptional regulation of transcription factor expression in Arabidopsis roots. PNAS 103, 6055-6060 (2006).

24. Durut, N. et al. A duplicated NUCLEOLIN gene with antagonistic activity is required for chromatin organization of silent 45D rDNA in Arabidopsis. Plant Cell 26, 1330-1344 (2014).

25. Sáez-Vásquez, J. \& Delseny, M. Ribosome biogenesis in plants: from functional 45 S ribosomal DNA organization to ribosome assembly factors. Plant Cell 31, 1945-1967 (2019).

26. Basu, U., Si, K., Warner, J. R. \& Maitra, U. The Saccharomyces cerevisiae TIF6 gene encoding translation initiation factor 6 is required for 60S ribosomal subunit biogenesis. Mol. Cell Biol. 21, 1453-1462 (2001).

27. Ho, J. H., Kallstrom, G. \& Johnson, A. W. Nmd3p is a Crm1p-dependent adapter protein for nuclear export of the large ribosomal subunit. J. Cell Biol. 151, 1057-1066 (2000).

28. Lo, K. Y. et al. Defining the pathway of cytoplasmic maturation of the 60S ribosomal subunit. Mol. Cell 39, 196-208 (2010).

29. Harnpicharnchai, P. et al. Composition and functional characterization of yeast $66 \mathrm{~S}$ ribosome assembly intermediates. Mol. Cell 8, 505-515 (2001).

30. Kater, L. et al. Visualizing the assembly pathway of nucleolar pre-60S ribosomes. Cell 171, 1599-1610 (2017)

31. Ma, C. et al. Structural snapshot of cytoplasmic pre-60S ribosomal particles bound by Nmd3, Lsg1, Tif6 and Reh1. Nat. Struct. Mol. Biol. 24, 214-220 (2017).

32. Matsuo, Y. et al. Coupled GTPase and remodelling ATPase activities form a checkpoint for ribosome export. Nature 505, 112-116 (2014). 
33. Gamalinda, M. et al. Yeast polypeptide exit tunnel ribosomal proteins L17, L35 and L37 are necessary to recruit late-assembling factors required for 27SB pre-rRNA processing. Nucleic Acids Res. 41, 1965-1983 (2013).

34. Morita, D. et al. Rpf2p, an evolutionarily conserved protein, interacts with ribosomal protein L11 and is essential for the processing of 27 SB Pre-rRNA to $25 \mathrm{~S}$ rRNA and the $60 \mathrm{~S}$ ribosomal subunit assembly in Saccharomyces cerevisiae. J. Biol. Chem. 277, 28780-28786 (2002).

35. Gnanasundram, S. V., Kos-Braun, I. C. \& Kǒs, M. At least two molecules of the RNA helicase Has1 are simultaneously present in pre-ribosomes during ribosome biogenesis. Nucleic Acids Res. 47, 10852-10864 (2019).

36. Browning, K. S. et al. Unified nomenclature for the subunits of eukaryotic initiation factor 3. Trends Biochem. Sci. 26, 284 (2001).

37. Jung, J. K. H. \& McCouch, S. Getting to the roots of it: genetic and hormonal control of root architecture. Front. Plant Sci. 4, 186 (2013).

38. Kaplan, F. et al. Exploring the temperature-stress metabolome of Arabidopsis. Plant Physiol. 136, 4159-4168 (2004).

39. Kaplan, F. et al. Transcript and metabolite profiling during cold acclimation of Arabidopsis reveals an intricate relationship of cold-regulated gene expression with modifications in metabolite content. Plant J. 50, 967-981 (2007).

40. Pagter, M. et al. Rapid transcriptional and metabolic regulation of the deacclimation process in cold acclimated Arabidopsis thaliana. BMC Genom. 18, 731 (2017).

41. Stitt, M. \& Hurry, V. A plant for all seasons: alterations in photosynthetic carbon metabolism during cold acclimation in Arabidopsis. Curr. Opin. Plant Biol. 5, 199-206 (2002).

42. Xiong, Y. et al. Glucose-TOR signalling reprograms the transcriptome and activates meristems. Nature 496, 181-186 (2013).

43. Dong, Y., Teleman, A. A., Jedmowski, C., Wirtz, M. \& Hell, R. The Arabidopsis THADA homologue modulates TOR activity and cold acclimation. Plant Biol. 21, 77-83 (2019).

44. Cheng, Z. et al. Small and large ribosomal subunit deficiencies lead to distinct gene expression signatures that reflect cellular growth rate. Mol. Cell 73, 36-47 (2019).

45. Li, L. et al. Protein degradation rate in Arabidopsis thaliana leaf growth and development. Plant Cell 29, 207-228 (2017).

46. Salih, K. J., Duncan, O., Li, L., Troesch, J. \& Millar, A. H. The composition and turnover of the Arabidopsis thaliana 80 S cytosolic ribosome. Biochem J. 477, 3019-3032 (2020).

47. Uesono, Y. \& Toh-e, A. Transient inhibition of translation initiation by osmotic stress. J. Biol. Chem. 277, 13845-13855 (2002).

48. Krokowski, D. et al. Characterization of hibernating ribosomes in mammalian cells. Cell Cycle 10, 2691-2702 (2011).

49. Van den Elzen, A. M. G., Schuller, A., Green, R. \& Seraphin, B. Dom34-Hbs1 mediated dissociation of inactive 80S ribosomes promotes restart of translation after stress. EMBO J. 33, 265-276 (2014).

50. Weis, B. L., Kovacevic, J., Missbach, S. \& Schleiff, E. Plant-specific features of ribosome biogenesis. Trends Plant Sci. 20, 729-740 (2015).

51. Krokowski, D., Tchórzewski, M., Boguszewska, A. \& Grankowski, N. Acquisition of a stable structure by yeast ribosomal P0 protein requires binding of P1A-P2B complex: in vitro formation of the stalk structure. Biochim. Biophys. Acta 1724, 59-70 (2005).

52. Krokowski, D. et al. Yeast ribosomal P0 protein has two separate binding sites for P1/P2 proteins. Mol. Microbiol. 60, 386-400 (2006).

53. Mitroshin, I. V., Garber, M. B. \& Gabdulkhakov, A. G. Investigation of structure of the ribosomal L12/P stalk. Biochemistry (Moscow) 81, 1589-1601 (2016).

54. Liljas, A. \& Sanyal, S. The enigmatic ribosomal stalk. Q. Rev. Biophys. 51, e12 (2018).

55. Holt, C. E. \& Schuman, E. M. The central dogma decentralized: new perspectives on RNA function and local translation in neurons. Neuron 80, 648-657 (2013).

56. Kim, Y., Zhang, H. \& Scholl, R. L. Two evolutionarily divergent genes encode a cytoplasmic ribosomal protein of Arabidopsis thaliana. Gene 93, 177-182 (1990).

57. Meinke, D. W. Genome-wide identification of EMBRYO-DEFECTIVE (EMB) genes required for growth and development in Arabidopsis. New Phytol. 226, 306-325 (2020).

58. Xue, S. \& Barna, M. Specialized ribosomes: A new frontier in gene regulation and organismal biology. Nat. Rev. Mol. Cell Biol. 13, 355-369 (2012).

59. Simsek, D. et al. The mammalian ribo-interactome reveals ribosome functional diversity and heterogeneity. Cell 169, 1051-1065 (2017).

60. Shi, Z. et al. Heterogeneous ribosomes preferentially translate distinct subpools of mRNAs genome-wide. Mol. Cell 67, 71-83 (2017).

61. Genuth, N. R. \& Barna, M. Heterogeneity and specialized functions of translation machinery: from genes to organisms. Nat. Rev. Genet. 19, 431-452 (2018).

62. Scholl, R. I., Ma, S. T. \& Ware, D. H. Seed and molecular resources for Arabidopsis. Plant Physiol. 124, 1477-1480 (2000).

63. Rosso, M. G. et al. An Arabidopsis thaliana T-DNA mutagenized population (GABI-Kat) for flanking sequence tag-based reverse genetics. Plant Mol. Biol. 53, 247-259 (2003).

64. Du, Z., Zhou, X., Ling, Y., Zhang, Z. \& Su, Z. AgriGO: a GO analysis toolkit for the agricultural community. Nucleic Acids Res. 38, W64-W70 (2010).

65. Benjamini, Y. \& Hochberg, Y. Controlling the false discovery rate: a practical and powerful approach to multiple testing. J. R. Stat. Soc. (Ser. B) 57, 289-300 (1995).

66. Erde, J., Loo, R. R. O. \& Loo, J. A. Enhanced FASP (eFASP) to increase proteome coverage and sample recovery for quantitative proteomic experiments. J. Proteome Res. 13, 1885-1895 (2014).

67. Swart, C., Martínez-Jaime, S., Gorka, M., Zander, K. \& Graf, A. Hit-Gel: Streamlining in-gel protein digestion for high-throughput proteomics experiments. Sci. Rep. 8, 8582 (2018).

68. The UniProt Consortium UniProt. the universal protein knowledgebase. Nucleic Acids Res. 45, D158-D169 (2017).

69. Zhang, W. et al. LFQuant: a label-free fast quantitative analysis tool for high-resolution LC-MS/MS proteomics data. Proteomics 12, 3475-3484 (2012).

70. Perez-Riverol, Y. et al. The PRIDE database and related tools and resources in 2019: Improving support for quantification data. Nucleic Acids Res. 47, D442-D450 (2019).

71. Barakat, A. et al. The organization of cytoplasmic ribosomal protein genes in the Arabidopsis genome. Plant Physiol. 127, 398-415 (2001).

72. Hummel, M. et al. Proteomic LC-MS analysis of Arabidopsis cytosolic ribosomes: Identification of ribosomal protein paralogs and re-annotation of the ribosomal protein genes. J. Proteom. 128, 436-449 (2015).

73. Sormani, R., Masclaux-Daubresse, C., Daniel-Vedele, F. \& Chardon, F. Transcriptional regulation of ribosome components are determined by stress according to cellular compartments in Arabidopsis thaliana. PLoS ONE 6, e28070 (2011).

74. Waltz, F. et al. Small is big in Arabidopsis mitochondrial ribosome. Nat. Plants 5, 106-117 (2019).

75. Rugen, N., Straube, H., Franken, L. E., Braun, H. P. \& Eubel, H. Complexome profiling reveals association of PPR proteins with ribosomes in the mitochondria of plants. Mol. Cell. Proteom. 18, 1345-1362 (2019). 


\section{Acknowledgements}

We acknowledge the research funding and facility provided by the Max Planck Society (Germany). We thank the University of Melbourne (Australia) for providing the Melbourne Research Scholarship to B.E.C. to support her PhD study. We thank Christopher Pries (MSc) for advice and support of transcriptome data preprocessing.

\section{Author contributions}

J.K., U.R., O.B.G., W.W.H.H., and B.E.C. conceived the original research plan. B.E.C. and O.B.G performed the experiments. O.B.G. preprocessed the transcriptome data. J.K., B.E.C. and O.B.G. analyzed the preprocessed transcriptome data. M.G. and A.S. generated and preprocessed the proteome data. J.K., F.M-S., and A.A.P.F. analyzed the preprocessed proteome data. J.K. and B.E.C. wrote the manuscript with contributions of all other authors.

\section{Funding}

Open Access funding enabled and organized by Projekt DEAL.

\section{Competing interests}

The authors declare no competing interests.

\section{Additional information}

Supplementary Information The online version contains supplementary material availlable at https://doi. org/10.1038/s41598-021-81610-z.

Correspondence and requests for materials should be addressed to J.K.

Reprints and permissions information is available at www.nature.com/reprints.

Publisher's note Springer Nature remains neutral with regard to jurisdictional claims in published maps and institutional affiliations.

(c) (i) Open Access This article is licensed under a Creative Commons Attribution 4.0 International License, which permits use, sharing, adaptation, distribution and reproduction in any medium or format, as long as you give appropriate credit to the original author(s) and the source, provide a link to the Creative Commons licence, and indicate if changes were made. The images or other third party material in this article are included in the article's Creative Commons licence, unless indicated otherwise in a credit line to the material. If material is not included in the article's Creative Commons licence and your intended use is not permitted by statutory regulation or exceeds the permitted use, you will need to obtain permission directly from the copyright holder. To view a copy of this licence, visit http://creativecommons.org/licenses/by/4.0/.

(C) The Author(s) 2021 